\title{
Cold-inducible promoter-driven knockdown of Brachypodium antifreeze proteins confers freeze sensitivity
}

1 Collin L. Juurakko ${ }^{1 *}$, Melissa Bredow ${ }^{1, \#}$, George C. diCenzo ${ }^{1}$, Virginia K. Walker ${ }^{1,2}$

$2{ }^{1}$ Department of Biology, Queen's University, Kingston, Ontario, Canada

$3 \quad{ }^{2}$ School of Environmental Studies, Queen's University, Kingston, Ontario, Canada

$4 *$ Correspondence:

5 Collin L. Juurakko

6 11cj10@queensu.ca

$7 \quad$ \# Current Address:

8 Department of Plant pathology and Microbiology, Iowa State University, Ames, Iowa, USA

9 Keywords: Brachypodium distachyon, cold acclimation, freeze tolerance, abiotic stress, 10 antifreeze proteins, ice-recrystallization inhibition

\section{Abstract}

The model forage crop, Brachypodium distachyon, has a family of ice recrystallization inhibition $(B d I R I)$ genes, which encode antifreeze proteins that function by adsorbing to ice crystals and inhibiting their growth. The genes were previously targeted for knockdown using a constitutive CaMV 35S promoter and the resulting transgenic Brachypodium showed reduced antifreeze activity and a greater susceptibility to freezing. However, the transgenic plants also showed developmental defects with shortened stem lengths and were almost completely sterile, raising the possibility that their reduced freeze tolerance could be attributed to developmental deficits. A cold-induced promoter

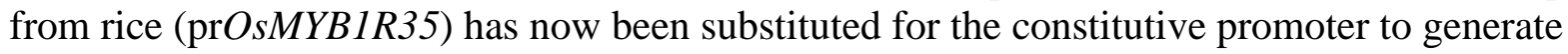
temporal miRNA-mediated Brachypodium antifreeze protein knockdowns. Although transgenic lines showed no apparent pleiotropic developmental defects, they demonstrated reduced antifreeze activity as assessed by assays for ice-recrystallization inhibition, thermal hysteresis, electrolyte leakage, leaf infrared thermography, and leaf damage after infection with an ice nucleating phytopathogen. Strikingly, the number of cold-acclimated transgenic plants that survived freezing at $-8{ }^{\circ} \mathrm{C}$ was reduced by half or killed entirely, depending on the line, compared to cold-acclimated wild type plants. Although these proteins have been studied for almost 60 years, this is the first unequivocal demonstration in any organism of the utility of antifreeze protein function and their contribution to freeze protection, independent of obvious developmental defects. These proteins are thus of potential interest in a wide range of biotechnological applications from accessible cryopreservation, to frozen product additives, to the engineering of transgenic crops with enhanced freezing tolerance.

\section{$31 \quad 1 \quad$ Introduction}

Increased freezing events and altered freeze-thaw cycles in response to climate change present major challenges to agriculture with single frosts costing billions of dollars (Witney and Arpaia, 1991; Sinha and Cherkauer, 2010; Smith and Katz, 2013; Kreyling, 2019; NOAA, 2021; Smith et al., 2021). In the field, the formation of ice at high sub-zero temperatures is initiated by ice nucleation active (INA+) bacteria and is a major driver of crop destruction (Snyder and Melo-Abreu, 
2005). Since they cannot escape low temperatures, many temperate climate plants have adopted a freeze tolerant strategy with some producing antifreeze proteins (AFPs) to help prevent freeze damage (Bredow and Walker, 2017; Juurakko et al., 2021a). In contrast, other organisms such as polar fish and temperate arthropods, which can escape low temperatures and find hibernacula, frequently adopt a freeze-avoidance strategy that can also employ AFPs, in this case to lower the freezing point relative to the melting point, also known as the thermal hysteresis (TH) gap (Duman, 2001; Bar Dolev et al., 2016; Kim et al., 2017). Despite the discovery of these proteins, first recognized almost 5 decades ago in Tenebrio beetles (Ramsay, 1964), until recently, there has been no formal evidence of their contribution to low temperature survival in any organism. This changed with the engineering of transgenic grass lines with knocked down expression of AFPs, resulting in greater freeze susceptibility (Bredow et al., 2016). However, important as these results were, it was worrying that the knockdowns were associated with other phenotypes including stunted growth and almost complete sterility, suggesting to naysayers that the greater freeze susceptibility could be due to unhealthy plants rather than the low AFP activity. To test that possibility, and to verify that AFPs do indeed make a crucial contribution to freeze survival, it was important that new transgenic lines be made that were unencumbered with detrimental phenotypes.

54

55

56

57

58

59

60

61

62

63

64

65

66

67

68

69

AFPs are also known as ice-binding proteins or ice-recrystallization inhibition (IRI) proteins. The plant freeze-tolerant overwintering strategy may be associated with AFPs that are characterised by low TH activity but a high IRI activity, which keeps ice crystals small even when the temperature fluctuates near $0^{\circ} \mathrm{C}$. This is important since ice forms in the apoplast, frequently due to INA+ bacteria, such as Pseudomonas syringae, gaining entry through stomatal openings, hydathodes, or wounding sites which themselves may result from surface tissue ice formation (Lindow et al., 1982; Ashworth and Kieft, 1995; Wisniewski and Fuller, 1999; Pearce and Fuller, 2001). Some plant AFPs can even attenuate the INA+ activity of $P$. syringae, possibly by binding to the bacterial ice nucleating proteins (INPs) and resulting in a modest lowering of the freeze temperature (Tomalty and Walker, 2014; Bredow et al., 2018). The apoplast has a lower solute concentration and thus freezes before other tissues. Thus, in order to combat catastrophic freezing, AFPs are produced and secreted to the apoplast (Griffith et al., 1992; Marentes et al., 1993; Hon et al., 1994; Hon et al., 1995; Antikainen et al., 1997; Bredow et al., 2016). The property of AFPs to irreversibly adsorb to ice crystals is key to membrane protection and explains why these proteins can be employed in both freeze-tolerant and freeze-avoidant strategies. In the absence of other ice management mechanisms, uncontrolled ice growth in the apoplast can lead to cellular death by dehydration through exclusion of solutes or the piercing of membranes, thus presenting the primary battleground between AFPs and ice (Lindow et al., 1982; Melo-Abreu et al., 2016).

Brachypodium distachyon (hereinafter Brachypodium) contains 7 genes encoding AFPs (BdIRI1-7). Their translation products are hydrolysed, likely in the apoplast, to generate two independent proteins, a leucine-rich repeat (LRR) protein that has not been characterised, and an AFP (Bredow et al., 2016). The BdIRI gene sequences are sufficiently similar so that a single miRNA could be designed to attenuate the translation of all 7 corresponding mRNAs with no obvious offtarget binding (Bredow et al., 2016). As noted, previously generated transgenic Brachypodium lines bearing the miRNA sequence, driven by the constitutive CaMV 35S promoter, were more susceptible to freeze damage than non-transgenic controls. Thus, although these experiments clearly connected AFPs with freeze protection, the lines also showed developmental deficits. We hypothesised that these secondary phenotypes were due to the constitutive promoter selected for those experiments, and that a cold-induced promoter, perhaps more similar to the native BdIRI promoters, would circumvent this problem and allow a fuller characterisation of newly-generated AFP knockdown lines. This has now been achieved and here we report an exploration of $B d I R I$ regulation as well as showing that the 
84 experimental temporal attenuation of AFP expression is inextricably linked to greater freeze

85 susceptibility.

\section{Methods}

\section{$87 \quad 2.1 \quad$ Bioinformatic Analysis}

BdIRI gene and protein sequences were retrieved from NCBI using up-to-date accessions (December 2020) using a BLAST search with the published proteins as queries (Bredow et al., 2016). The lack of data for the identification of suitable low-temperature inducible promoters in Brachypodium prompted the selection of the 1961 bp promoter associated with the rice, Oryza sativa, gene OsMYB1R35, which is induced in its host plant after cold stress (Li et al., 2017). The sequence was retrieved from the publicly available $O$. sativa genome on NCBI (accessed October 2017) based on the primer sequences described elsewhere (Li et al., 2017). The $1961 \mathrm{bp}$ fragment was synthesized by GeneART (Thermo Fisher Scientific, Waltham, MA) with appropriate flanking restriction enzyme recognition sites. Conceptually translated $B d I R I$ sequences were aligned and phylogenies prepared using the Clustal Omega Multiple Sequence Alignment tool

(https://www.ebi.ac.uk/Tools/msa/clustalo/) (Sievers et al., 2011). Inter-domain hydrolytic cleavage sites in the amino acid sequence corresponding to the BdIRIs were predicted using the ExPASy

100 PeptideCutter tool (https://web.expasy.org/peptide_cutter/; Gasteiger et al. 2005). Chromosomal positioning of the BdIRI genes was determined using NCBI's genome browser. InterProScan (Version 83.0; https://www.ebi.ac.uk/interpro/) was used for the in silico prediction of the LRR and AFP domains in the BdIRI translated sequences (Blum et al., 2021). The Phyre 2 Protein Fold Recognition Server (http://www.sbg.bio.ic.ac.uk/ phyre2/) was used for sequence-based homologous protein structure prediction of the corresponding BdIRI domains (Kelley et al., 2015). The SignalP 5.0 Server (http://www.cbs.dtu.dk/services/SignalP/) was used to forecast putative amino-terminal secretory signal peptide sequences (Armenteros et al., 2019). The DeepLoc 1.0 eukaryotic protein subcellular localization predictor (http://www.cbs.dtu.dk/services/DeepLoc-1.0/) was used to confirm the subcellular localization of proteins secreted to the apoplast (Armenteros et al., 2017).

\subsection{Prediction of miRNA Targets and Analysis of Regulatory Elements}

Identification of post-transcriptional regulation via endogenous miRNAs was performed on (http://plantgrn.noble.org/psRNATarget/) (Dai et al., 2018). The tool was used with data from miRBase (Release 21, June 2014) (Griffiths-Jones, 2004; Griffiths-Jones et al., 2006; Griffiths-Jones et al., 2007; Kozomara and Griffiths-Jones, 2010; Kozomara and Griffiths-Jones, 2014; Kozomara et al., 2019).

Upstream sequences to the translational start codons of selected genes were retrieved from NCBI (B. distachyon genome assembly v3 2020) in addition to the promoter sequence of prOsMYB1R35 from O. sativa rice (Li et al., 2017). Sequences were submitted to PlantCARE (http://bioinformatics.psb.ugent.be/webtools/plantcare/html/) (Lescot et al., 2002) for cis-regulatory element prediction and analysis. Raw output files were converted to text and input into excel where they were compiled for a comparative analysis of cis-regulatory elements among the putative promoter sequences used. Cis-regulatory elements specifically related to cold signalling and cold stress were manually annotated for putative promoter sequences of all BdIRIs and OsMYBIR35, designated as prBdIRIs 1-7 and prOsMYBIR35, respectively.

\subsection{Plasmids Construction and Brachypodium Transformation}


The GeneART pUC plasmid containing the prOsMYB1R35 promoter sequence was transformed into Escherichia coli DH5 $\alpha$ cells (Thermo Fisher Scientific). The promoter was liberated from purified plasmid using BamHI and BglII restriction enzyme digests for the 5' and 3' end, respectively. It was then ligated into the plant expression plasmid pCambia1380 (Marker Gene Technologies Inc., Eugene, OR, USA) and transformed into E. coli DH5 $\alpha$. A plasmid bearing a sequence corresponding to the miRNA (Bredow et al., 2016) with a 5' BglII site, was amplified by polymerase chain reaction (PCR) and a 3' SpeI restriction site was added with the use of appropriate primers. Similarly, the sequence encoding enhanced green fluorescent protein (eGFP; GenBank Accession no. U57607) was PCR-amplified and 5' BglII and 3' SpeI restriction sites were added. The pCambia1380 with the prOsMYBIR35 insert was then digested using BglII and SpeI enzymes and the purified amplified products, miRNA and eGFP, were ligated separately to create the plasmids pCambia1380:prOsMYB1R35:miRNA and pCambia1380:prOsMYB1R35:eGFP, respectively. These plasmids were independently transformed into $E$. coli DH5 $\alpha$ and the veracity of their sequence subsequently confirmed by Sanger sequencing (CHU de Québec-Université Laval, Quebec City, QC, CA). DNA from the verified plasmids was isolated, purified and transformed into Agrobacterium tumefaciens (AGL1, Invitrogen, Carlsbad, CA, USA) (hereinafter, Agrobacterium). Again, the authenticity of the target sequences was checked by Sanger sequencing. AGL1 cells containing the confirmed plasmids were then used for transformation into Brachypodium.

Transgenic Brachypodium lines were generated using a modified method from Fursova et al. (2012) as follows. Transformed Agrobacterium cultures (50 mL), were grown in Luria Bertani (LB) broth containing $50 \mathrm{mg} \mathrm{L}^{-1}$ kanamycin to an $\mathrm{OD}_{600}$ of 1 , pelleted at $5000 \times g$ for $10 \mathrm{~min}$, and washed in equal volumes of 2-(N-morpholino)ethanesulfonic acid (MES) infiltration buffer (10 mM MES, 10 $\mathrm{mM} \mathrm{MgCl}_{2}, \mathrm{pH}$ 5.6). Pellets were resuspended in $50 \mathrm{~mL}$ of infiltration buffer containing $50 \mu \mathrm{M}$ acetosyringone, $0.01 \%$ Silwet-L77 organosilicone surfactant, and leaf extracts containing phenolic metabolites to initiate efficient Ti plasmid transfer made from Australian tobacco, Nicotiana benthamiana leaves, rather than Nicotiana tabacum (Fursova et al., 2012). To prepare the tobacco extracts, up to $50 \mathrm{~g}$ of leaf tissue was harvested from six-week-old plants, cut into $1-3 \mathrm{~cm}^{2}$ squares, and incubated in $300 \mathrm{~mL}$ of infiltration buffer for $2 \mathrm{~h}$. The liquid was removed by pressing a sterile beaker of slightly smaller diameter into the slurry and subsequently filter-sterilising the recovered liquid through 0.22-micron syringe filters (Thermo Fisher Scientific). Acetosyringone and surfactant were added following filter sterilisation and used to resuspend the washed Agrobacterium pellet. In parallel, mature wild type seeds (50 per trial) were harvested, surface-sterilised as described, and aseptically trimmed using a sterile scalpel to remove the upper quarter of the seed where the awn had been attached. The lower portion of the sterilised and trimmed seeds, with exposed embryos, were immediately added to the now-primed Agrobacterium culture and co-cultivated for $30 \mathrm{~h}$ at $21{ }^{\circ} \mathrm{C}$ with shaking at $200 \mathrm{rpm}$, in the dark.

Following co-cultivation, seeds were washed in infiltration buffer alone and plated on Linsmaier and Skoog (LS) agar (Phytotech Labs, Lenexa, KS, USA) containing $225 \mathrm{mg} \mathrm{L}^{-1}$ timentin, a wide-spectrum antibacterial, in order to kill residual Agrobacterium. The plates were sealed with micropore tape and put at Brachypodium standard growth conditions (see below). One week following successful germination, surviving $\mathrm{T}_{0}$ generation seedlings were sown to soil and grown. Hygromycin selection media was not used, since initial experiments suggested that this treatment was often detrimental to the seedlings. DNA was subsequently extracted from $\mathrm{T}_{0}$ plants using Monarch DNA extraction kits (New England Biolabs, Ipswich, MA, USA) and sequence confirmed (CHU de Québec-Université Laval, Quebec City, QC, Canada). Once T 0 plants were brought to senescence, seeds were counted and harvested. Seeds from these plants (10 per line) were sterilised and germinated on LS agar with hygromycin. Selected, germinated seeds were sown in soil and brought 
174

175

176

177

178

179

180

181

182

183

184

185

186

187

188

189

190

191

192

193

194

195

196

197

198

199

200

201

202

203

204

205

206

207

208

209

210

to senescence representing the $\mathrm{T}_{1}$ generation. After collection, seeds ( 24 per line) were sterilised and germinated on LS agar without antibiotics. These $\mathrm{T}_{2}$ generation plants were used for rapid genotyping (see below) to determine homozygosity for the $\mathrm{T}_{3}$ generation without the need for selection.

\subsection{Genotyping Brachypodium Transgenic Lines}

Genotyping was done using a modified method from Ben-Amar et al. (2017). Briefly, seeds (24 per line) were sterilised, germinated, and sown in soil. Leaf tips ( $\sim 2 \mathrm{~mm}$ long) were cut at two weeks and placed in individual wells of 96 well PCR plates, each holding $50 \mu \mathrm{L}$ of TE buffer at $\mathrm{pH}$ 8.0. The leaf tissue was gently ground using sterile fine-tip forceps, which were washed in $70 \%$ ethanol between samples. Once all samples were homogenised, plates were incubated at $60{ }^{\circ} \mathrm{C}$ for 10 min and then gently vortexed. Extracted samples $(2 \mu \mathrm{L})$ from each line were combined into a $1.5 \mathrm{~mL}$ tube and gently vortexed, and from this pool containing extracts from each plant, $1 \mu \mathrm{L}$ was used as the DNA template for a PCR screen of the insert. Lines contributing to the pooled extracts showing an amplified DNA band of an appropriate size after gel electrophoresis were then selected for individual sample screening. For this, $1 \mu \mathrm{L}$ of sample extracts from individual plants were used as PCR templates, with lines showing a positive insert band for every individual considered to be homozygous. The reference gene, S-adenosylmethionine decarboxylase $(S A M D C)$ was used as a PCR control.

\subsection{Western Blot Analysis}

Western blot analysis was conducted on leaf samples from three-week-old wild type (Bd21) plants or transgenic plants maintained under standard conditions (non-acclimated; NA) or transferred to $4{ }^{\circ} \mathrm{C}$ for $48 \mathrm{~h}$ (cold-acclimated; CA, see details below). These plants were obtained by using seeds from wild type or transgenic prOsMYB1R35:eGFP plants. Subsequent to surface sterilisation, the seeds were germinated on selection media with $50 \mathrm{mg} \mathrm{mL}^{-1}$ hygromycin B (BioShop Canada Inc., Burlington, ON, Canada). After sowing on potting soil, they were grown for three weeks before harvest of $200 \mathrm{mg}$ of leaf tissue from each plant. After freezing with liquid nitrogen and grinding to a fine powder using a sterile micro pestle, the samples were resuspended in extraction buffer $(5 \mathrm{mM}$ DTT, $1 \%$ Sigma P9599 protease inhibitor, 0.1\% Igepal, $2 \mathrm{mM} \mathrm{NaF}, 1.5 \mathrm{mM}$ activated $\mathrm{Na}_{3} \mathrm{VO}_{4}, 0.5$ $\mathrm{M}$ Tris- $\mathrm{HCl} \mathrm{pH} 7.5,10 \%$ glycerol, $0.15 \mathrm{M} \mathrm{NaCl}$ ) and shaken at $150 \mathrm{rpm}$ at $4{ }^{\circ} \mathrm{C}$ for $4 \mathrm{~h}$. Following centrifugation $\left(13,000 \times \mathrm{g}\right.$ at $4{ }^{\circ} \mathrm{C}$ for $\left.30 \mathrm{~min}\right)$, the protein concentration in the supernatants was estimated (Bradford reagent, Thermo Fisher Scientific) and then diluted so that samples were equivalent. Subsequently, $100 \mu \mathrm{L}$ of the samples were denatured by the addition of $50 \mu \mathrm{L}$ of Laemmli Sample Buffer (45\% glycerol, 10\% SDS, 0.5 M Tris pH 6.8, 0.045\% w/v bromophenol blue, $0.006 \% 1 \mathrm{M} \mathrm{DTT}$ ) and boiled for $5 \mathrm{~min}$. Samples were run using a semi-dry transfer apparatus (Bio-Rad Laboratories, Hercules, CA, USA) following the manufacturer's recommended protocols. The membrane was blocked for $1 \mathrm{~h}$ using a 5\% (w/v) skim milk powder in Tris-buffered saline with $0.1 \%$ Tween $^{\circledR} 20$ detergent (TBST) while shaking at room temperature.

GFP was detected on the membranes using anti-GFP mouse-IgG monoclonal (clones 7.1 and 13.1) antibody (Roche, Basel, Switzerland) in a 1/500 dilution in 5\% (w/v) skim milk powder TBST solution with gentle shaking overnight at $4{ }^{\circ} \mathrm{C}$ in the dark. The secondary antibody, anti-mouse IgG peroxidase-conjugated (Sigma-Aldrich, St. Louis, MO, USA) was used in a 1/4000 dilution in the same skim milk-TBST solution with gentle shaking at room temperature for $1 \mathrm{~h}$. Coomassie brilliant blue stained ribulose-1,5-bisphosphate carboxylase-oxygenase (better known as RuBisCO) large chain (RbcL) was used as a $55 \mathrm{kDa}$ loading control. Blots were washed with TBST buffer three times 
for 10 min each time and inserted between acetate transparency sheets and imaged on a ChemiDoc Touch Imaging System (Bio-Rad Laboratories) using Immobilon western chemiluminescent HRP substrate (MilliporeSigma, Boston, MA, USA). Western blot analysis was done using Image Lab Software (Bio-Rad Laboratories). Purified recombinant eGFP was used as a positive control and western blots were repeated in triplicate.

\section{3}

\subsection{Plant Material and Growth Conditions}

Brachypodium seeds of an inbred line, ecotype Bd21 (RIKEN, Tokyo, Japan), were prepared by soaking in sterile water for $1 \mathrm{~h}$ followed by careful removal of the lamella, awn, and any remaining appendages still attached to the harvested floret. Seeds were washed in a $40 \%$ bleach, $0.04 \% \mathrm{w} / \mathrm{v}$ Silwet-L77 solution for 4 min followed by a 2 min wash in $70 \%$ ethanol and 4 washes in sterile water and finally dried with filter paper soaked in $100 \%$ ethanol. Seeds were transferred to LS agar plates using sterilised forceps and the plates were subsequently sealed using sterile micropore tape. All seed work was done in a UV sterilised laminar flow hood. Plates were subsequently wrapped in aluminium foil and placed at $4{ }^{\circ} \mathrm{C}$ to synchronise germination for four days.

After this time, plates were moved to a climate-controlled growth chamber (Conviron CMP4030, Controlled Environments Limited, Winnipeg, MB, Canada) at standard Brachypodium growth conditions of $70 \%$ relative humidity and $24 \mathrm{~h}$ cycles of $20 \mathrm{~h} \mathrm{light}\left(\sim 150 \mu \mathrm{mol} \mathrm{m} \mathrm{s}^{-1}\right)$ at 24 ${ }^{\circ} \mathrm{C}$ followed by $4 \mathrm{~h}$ with no light at $18{ }^{\circ} \mathrm{C}$. After one week, seeds were transplanted to pots filled with moist potting soil and then fertilised bi-weekly using 10-30-20 Plant-Prod MJ Bloom (Master PlantProd, Brampton, ON, Canada). Prior to assaying, CA plants were moved to a separate chamber (Econair GC-20, Ecological Chambers Inc., Winnipeg, MB, Canada) maintained at $4{ }^{\circ} \mathrm{C}$ where they were subjected to a shortened day cycle of $6 \mathrm{~h}$ of light $\left(\sim 150 \mu \mathrm{mol} \mathrm{m} \mathrm{m}^{-2} \mathrm{~s}^{-1}\right)$ and $20 \mathrm{~h}$ of darkness for $48 \mathrm{~h}$. NA plants remained at standard conditions.

\subsection{Crude Lysate and Apoplast Extract Preparations}

AFP activity was assayed in extracts prepared as described previously (Bredow et al., 2016). After CA, $50 \mathrm{mg}$ of leaf tissue was taken from the three-week-old plants and ground to a fine powder using a sterile pestle after being flash frozen with liquid nitrogen. After suspension in $400 \mu \mathrm{L}$ of NPE buffer (25 mM Tris, $10 \mathrm{mM} \mathrm{NaCl}, \mathrm{pH} 7.5$, EDTA-free protease inhibitor tablets), the slurry was shaken for $4 \mathrm{~h}$ at $4{ }^{\circ} \mathrm{C}$ in the dark on a GyroMini nutating mixer (Labnet International, Edison, NJ, USA). Samples were centrifuged at $13,000 \times g$ for $5 \mathrm{~min}$ and placed at $4{ }^{\circ} \mathrm{C}$ for $5 \mathrm{~min}$ and then the centrifugation and the incubation were repeated. The supernatant was transferred to $1.5 \mathrm{~mL}$ tubes and centrifuged again $(13,000 \times g$ for $5 \mathrm{~min})$ before returning to $4{ }^{\circ} \mathrm{C}$. Protein concentration was estimated using a Nanodrop One (Thermo Fisher Scientific; using a mass extinction coefficient $\left(\varepsilon_{1 \%}\right)$ of 10 at $280 \mathrm{~nm}$ for $10 \mathrm{mg} \mathrm{mL}^{-1}$ with a baseline correction at $370 \mathrm{~nm}$, as recommended by the manufacturer). Readings were performed in triplicate for each sample, with samples routinely diluted to $1 \mathrm{mg} \mathrm{mL}^{-1}$ unless stated otherwise.

Apoplast extracts were prepared as previously described (Pogorelko et al., 2011) with minor modifications. Briefly, $0.5 \mathrm{~g}$ of leaf tissue of three-week-old NA and CA wild type and knockdown lines were collected. Leaf tissue was sliced with a sterile scalpel into $1 \mathrm{~cm}$ segments and placed vertically into a $10 \mathrm{~mL}$ syringe with the tip sealed with parafilm. Chilled, $4{ }^{\circ} \mathrm{C}$ extraction buffer $(5$ $\mathrm{mL}$ of $25 \mathrm{mM}$ Tris- $\mathrm{HCl}, 50 \mathrm{mM}$ EDTA, $150 \mathrm{mM} \mathrm{MgCl}_{2}, \mathrm{pH} 7.4$ ) was added and the syringe was placed under vacuum for $1 \mathrm{~min}$, four separate times, until bubbles ceased and leaves were fully infiltrated and darker in appearance. Excess buffer was drained, and leaf tissue was transferred to a 3 
$\mathrm{mL}$ syringe. The syringe, without the plunger, was then placed into a $15 \mathrm{~mL}$ conical tube and centrifuged at $1000 \times \mathrm{g}$ for $10 \mathrm{~min}$ at $4{ }^{\circ} \mathrm{C}$. Apoplast fluid was collected at the bottom of the conical tube and transferred to a fresh $1.5 \mathrm{~mL}$ microcentrifuge tube. Protein concentration was estimated using a Synergy H1 microplate reader (BioTek Instruments, Inc., Winooski, VT, USA) with a Take3 Micro-Volume Plate (BioTek Instruments) at $\mathrm{A}_{280}$, as described for the lysates. Samples were normalised and diluted as described prior to assaying. All work was carried out at $4{ }^{\circ} \mathrm{C}$.

\subsection{AFP assays}

To assess IRI activity, a modified "splat" assay was performed as previously described

Samples for TH assays were prepared as described (Bredow et al., 2020), but with $200 \mathrm{mg}$ of
(Millipore) were used to (Bredow et al., 2017). Briefly, samples $(10 \mu \mathrm{L})$ were pipetted $1 \mathrm{~m}$ above a glass cover slip, equilibrated on an aluminium block that had been chilled with dry ice for $1 \mathrm{~h}$, to ensure the formation of a thin layer of small ice crystals prior to transfer into a hexane-containing bath for annealing maintained at $-6{ }^{\circ} \mathrm{C}$. Images were captured through cross-polarising films at $10 \mathrm{x}$ magnification, immediately after transfer to the bath and again after annealing for $18 \mathrm{~h}$. Lysates and apoplast extracts were subjected to a standardised dilution series and assays were done a minimum of three times for all samples. concentrate supernatants 4 -fold after centrifugation $(13,000 \times \mathrm{g}$ for $10 \mathrm{~min})$. TH was determined on a nanoliter osmometer as described (Middleton et al., 2014). Ice crystal morphology was recorded during the $\mathrm{TH}$ assays with images captured using a microscope video camera at 50x magnification and in triplicate.

\subsection{Electrolyte Leakage, Infrared Thermography, Whole Plant Freezing Assays and Infections}

Electrolyte leakage assays were conducted as described (Bredow et al., 2016). Briefly, leaf tips ( $4 \mathrm{~cm}$ long) were excised from three-week-old plants and placed individually into sterilised glass test tubes containing $100 \mu \mathrm{L}$ of deionized water with the wounded end up and the tip submerged in the water. Sterilised foam plugs were used to ensure that the leaf tips remained submerged. One set of control tubes contained leaf tips from individual, numbered plants and were kept at $4{ }^{\circ} \mathrm{C}$ in the dark. The second companion set of tubes with leaves were placed in a programmable circulating ethylene glycol temperature-controlled bath set at $0{ }^{\circ} \mathrm{C}$. The temperature was ramped down from $0{ }^{\circ} \mathrm{C}$ to $-1{ }^{\circ} \mathrm{C}$ over $30 \mathrm{~min}$ and then a single ice chip was added to initiate ice crystal growth, with the temperature then lowered $1{ }^{\circ} \mathrm{C}$ every $15 \mathrm{~min}$ until the final freezing temperature of $-10{ }^{\circ} \mathrm{C}$ was reached. The tubes were then placed at $4{ }^{\circ} \mathrm{C}$ in the dark with the control tubes and left overnight. After transfer of the tube contents to $50 \mathrm{~mL}$ centrifuge tubes containing 25 $\mathrm{mL}$ of deionized water, they were shaken horizontally on a G2 Gyrotory Shaker (New Brunswick Scientific, Edison, NJ, USA) at $150 \mathrm{rpm}$ for $18 \mathrm{~h}$ in the dark at room temperature. Initial conductivity $\left(\mathrm{C}_{\mathrm{i}}\right)$ was measured using an Oakton CON 510 conductivity metre (OAKTON Instruments, Vernon Hills, IL, USA) prior to autoclaving the samples for $45 \mathrm{~min}$. Samples were cooled to room temperature overnight and final conductivity $\left(\mathrm{C}_{\mathrm{f}}\right)$ was then measured. The percentage of electrolyte leakage was calculated using $\left(100 \times \mathrm{C}_{\mathrm{i}} / \mathrm{C}_{\mathrm{f}}\right)$. The assay was performed in triplicate using leaves from 10 individual plants per line and condition.

To visualise ice propagation in leaves and the influence of AFPs, infrared thermography was used, which detects emitted infrared energy provided that the objects have sufficiently high levels of 
304

305

306

307

308

309

310

311

312

313

314

315

316

317

318

319

320

321

322

323

324

325

326

327

328

329

330

331

332

333

334

335

336

337

338

339

340

341

342

343

344

345

346

347

emissivity over background. In plants, emissivity ratings are typically in the range of 0.98 (López et al., 2012; Chen, 2015). To enhance the contrast, household aluminium foil was used as a background because of its low emissivity of 0.05 (Qin et al., 2017). The FLiR One Pro - iOS (FLIR Systems, Wilsonville, OR, USA) with Vernier Thermal Analysis Plus application (Apple App Store) (Vernier Software and Technology, Beaverton, OR, USA) was used to capture thermography data.

Brachypodium leaves of equal length $(\sim 2.5 \mathrm{~cm})$ were freshly excised from CA three-week-old plants and placed on a stage lined with aluminium foil touching the surface of a circulating ethylene glycol bath set at $1{ }^{\circ} \mathrm{C}$. Leaf tissue was annealed for 30 min before distilled water $(10 \mu \mathrm{L})$ was pipetted onto the wounded end of the leaf and subsequently sterile ice chips of equal size were added to each sample to initiate nucleation. The temperature was then lowered to $-10{ }^{\circ} \mathrm{C}$ at $0.01{ }^{\circ} \mathrm{C} \mathrm{sec}^{-1}$.

Temperature measurement points were set with the software for each leaf $\sim 1 \mathrm{~cm}$ from the wound and recorded as freezing progressed. Data was analysed using Logger Pro (version 3.14) (Vernier Software and Technology). The assays were performed in triplicate.

Assessment for whole plant freeze survival was modified from previous methods (ColtonGagnon et al., 2014; Bredow et al., 2016; Mayer et al., 2020). Brachypodium seeds were sterilised and sown with 10 seeds per line and condition, and evenly spaced apart in 4" x 6" x 3" pots, as described in plant growth conditions. Seeds were then stratified in darkness at $4{ }^{\circ} \mathrm{C}$ for 4 days to synchronise germination. Seedlings were grown for two weeks at standard, previously described conditions. The pots were then transferred to another climate-controlled chamber (Econair GC-20; Ecological Chambers Inc.) where plants were exposed to $-1^{\circ} \mathrm{C}$ for $12 \mathrm{~h}$ before the lights were turned off and temperature was ramped down at a rate of $1{ }^{\circ} \mathrm{C}$ per hour until $-8{ }^{\circ} \mathrm{C}$, and after which the temperature was returned to $4{ }^{\circ} \mathrm{C}$ and the lighting resumed $\left(\sim 150 \mu \mathrm{mol} \mathrm{m}{ }^{-2} \mathrm{~s}^{-1}\right)$ for $24 \mathrm{~h}$. After recovery, plants were returned to standard conditions for 2 weeks, survival was recorded, and images were captured. Survival assays were repeated in triplicate.

Infections at high subzero temperatures were assayed using liquid cultures of $P$. syringae pv. syringae B728A, a pathovar with ice nucleation activity (Feil et al., 2005). The bacteria were cultured while shaking at $28{ }^{\circ} \mathrm{C}$ to $\mathrm{OD}_{600}=0.6-1.0$. They were then placed at $4{ }^{\circ} \mathrm{C}$ for two days while shaking to increase INP production and resuspended in $10 \mathrm{mM} \mathrm{MgCl}_{2}$ and diluted to an $\mathrm{OD}_{600}$ of 0.2 , corresponding to an approximate concentration of $1 \times 10^{8}$ colony forming units (CFU) $\mathrm{mL}^{-1}$. Simultaneously, three-week-old plants were $\mathrm{CA}$ at $4{ }^{\circ} \mathrm{C}$ for two days. Extra care was taken to ensure plants and cultures remained separated. Leaves of equal size and length were aseptically removed and the wound was dipped in the bacterial cultures. Leaves were then incubated at $-3{ }^{\circ} \mathrm{C}$ in an enclosed temperature regulated chamber for $12 \mathrm{~h}$, assessed for evidence of disease including water soaking and cell death and then allowed to recover at $4{ }^{\circ} \mathrm{C}$ in the dark with reassessment at $24 \mathrm{~h}, 48 \mathrm{~h}$, and one week. Preliminary experiments involved plants grown at standard conditions for three weeks with NA and CA plants sprayed with culture as well as cut leaves exposed to wound dipping as described. Whole plants and leaf tissue preliminary infections were carried out at $-3{ }^{\circ} \mathrm{C}$ and recovered as described with separate plants and leaf tissue maintained at standard Brachypodium conditions following infection as controls. All assays were repeated a minimum of three times.

\section{Results}

\subsection{BdIRI Gene Analysis}

The number of predicted full-length IRI genes in Brachypodium database has changed as genome assembly quality and annotation tools have improved. Based on the v3 RefSeq annotation (GCF_000005505.3), Brachypodium is predicted to encode six full-length IRI genes (BdIRI1, 
BdIRI3, BdIRI4, BdIRI5, BdIRI6 and BdIRI7) and one gene (BdIRI2) encoding a truncated protein with an intact LRR domain but with no AFP domain. Alignments of all amino acid sequences corresponding to BdIRII-7 using Clustal Omega from the v3 assembly showed annotated apoplast localization signal sequences, LRR motifs of LxxL where x represents a non-conserved residue, putative protease hydrolysis sites, as well as AFPs (except for BdIRI2) with ice-binding motifs of $\mathrm{NxVxG} / \mathrm{NxVxxG}$ where $x$ represents an outward-facing residue of the beta-barrel structure (Figure $\mathrm{S} 1$ ). The genes are found in three gene clusters on chromosome five (Figure S2). The organisation shows an adjacent position of two to three genes, with transcription in the same direction within each cluster, and flanked by distinct genes including some that may be involved in epigenetic regulation. It is possible that adjacent BdIRI loci are similarly spatio-temporally or developmentally regulated. For example, BdIRI3 and BdIRI4 are adjacent in the genome, and peptides corresponding to the AFP domain of both of these genes were identified after mass spectrometry of CA Brachypodium leaves (Bredow et al., 2016).

To obtain insight into the regulation of the BdAFP genes to better devise a knockdown strategy that targeted AFP expression only after exposure of the plant to low temperatures, sequences upstream of the translational start site of the BdIRIs, as well as the promoter region from a known cold-inducible gene from rice, prOsMYBR1R35 (Figure 1; Li et al., 2017), were examined for putative cis-acting regulatory elements (CAREs) using PlantCARE (Figure 1; Table S1). All sequences were found to contain cis-elements including canonical promoters or enhancers such as TATA-boxes and CAAT-boxes. Cold response-related and drought-resistant (CRT/DRE) core motifs (CCGAC), which are cis-elements involved in low-temperature stress responses, were associated with all the promoters. Additional motifs associated with stress and low-temperatures including various abscisic acid-responsive elements (ABREs), low-temperature response elements (LTREs), inducer of cold or C-repeat binding factor expression 1 CBF expression 1 (ICEr1), drought response elements (DREs), and the WRKY stress transcription factors recognition W-box motifs were all found, and these have been annotated (Figure 1). The cold-inducible rice promoter shared with the $B d$ AFP promoters multiple CRT/DRE motifs in addition to other elements involved in low temperature regulation and thus it was surmised to be suitable to drive expression of the inhibitory miRNA.

Plant gene expression can be regulated by miRNAs both transcriptionally (Yang et al., 2019) and post-transcriptionally (Jones-Rhoades et al., 2006; Mallory and Vaucheret, 2006; Bertolini et al., 2013; Zhang, 2015), with Brachypodium known to transcribe miRNAs as part of its cold-stress response (Zhang et al., 2009). Among monocots, Brachypodium and rice have the highest number of annotated miRNAs with 525 and 713 (O. sativa) in the miRBase database (Release 21). Again, to inform the knockdown strategy, all 7 BdIRI genes, as well as the rice promoter, prOsMYBR1R35, were analysed for potential miRNA binding sites. Within the BdIRI coding regions, the majority of miRNAs (14/28), belong to the bdi-miR395 family which have homologs in rice as well as Arabidopsis (Zhang et al., 2009). This miRNA family is stress-regulated with members known to target disease resistant proteins (Jones-Rhoades et al., 2004; Fujii et al., 2005; Baev et al., 2011; Lv et al., 2016). Other miRNAs from the miR169 family had 6 putative targets in BdIRI2 and BdIRI5 and are predicted to be involved in the plant oxidative stress response ( $\mathrm{Lv}$ et al., 2016). Likewise, other miRNAs are predicted to regulate multiple BdIRIs including bdi-miR7717a-5p on BdIRI2 and BdIRI3 and bdi-miR5055 on BdIRI2, BdIRI3, BdIRI5, and BdIRI6, suggesting that BdIRIs can be regulated by common miRNAs, in addition to other miRNAs that target individual BdIRI transcripts. For example, BdIRI4, -3 and -1 are clustered on the chromosome but only BdAFP isoforms 3 and 4 were found after mass spectrophotometric analysis of leaves (Bredow et al. 2016), suggesting that the lack of the $B d$ AFP isoform 1 in that tissue may be due to miRNA regulation by bdi-miR $159 \mathrm{~b}-3 \mathrm{p} .2$ 
395

396

397

398

399

400

401

402

403

404

405

406

407

408

409

410

411

412

413

414

415

416

417

418

419

420

421

422

423

424

425

426

427

428

429

430

431

432

433

434

435

436

437

with a binding site in the $B d I R I I$ transcript but not in the other BdIRI transcripts. In total, the algorithm indicated that all of the BdIRIs had at least one predicted miRNA target in the corresponding transcript and all but BdIRI4 showed miRNA binding sites upstream of the coding region (Table S2). Notably the 1961 bp rice promoter region also showed multiple Brachypodium miRNA target sites, as did sequences corresponding to BdIRII, $-2,-5$, and -7 gene promoters (Table S2). The observation that the low temperature-regulated promoter regions from both species shared some Brachypodium miRNA target sites (e.g. miR1583 and miR5174d), again suggested that the choice of this rice promoter to drive expression of the miRNA in BdIRI knockdown lines was likely appropriate.

\subsection{Promoter Function and Developmental Phenotypes}

In an attempt to curtail pleiotropic effects that might have been due to the constitutive expression of the miRNA in previous knockdown constructs (Bredow et al., 2016), CaMV 35S was substituted with the cold-induced rice promoter. After construction of the plasmids, Brachypodium was successfully transformed using a seed cut method (modified from Fursova et al., 2012), which from a total of 150 seeds, yielded three and two PCR-positive transformants for the miRNA and eGFP constructs, respectively. The rapid genotyping method (Ben-Amar et al., 2017) proved effective and overall, $65 \%$ of the recovered $\mathrm{T}_{1}$ generation were resistant to the hygromycin-selective media.

Once homozygous lines were selected, the ability of the heterologous rice promoter to direct transcription was assessed using western blots with transgenic control Brachypodium bearing the prOsMYB1R35:eGFP construct (Figure S3). No bands corresponding to GFP were detected in NA or CA wild type extracts, nor in NA prOsMYB1R35:eGFP leaves. However,, CA prOsMYB1R35:eGFP extracts showed a band at $26 \mathrm{kDa}$ that co-migrated with purified GFP. This demonstrates the successful expression of a marker protein in Brachypodium, driven by the OsMYB1R35 promoter from $O$. sativa.

Plants bearing the rice promoter ligated to the miRNA sequence appeared to develop similarly to wild type and showed normal phenotypes with respect to height and seed production (Figure S4). Two homozygous lines were identified and designated prOmiRBdIRI-1e and prOmiRBdIRI-3c. The prOmiRBdIRI-1e and prOmiRBdIRI-3c lines were $25.8 \pm 4.07 \mathrm{~cm}$ and $21.86 \pm 4.37 \mathrm{~cm}$, and set $106.2 \pm 29.9$ and $99.5 \pm 32.6$ seeds, respectively, which was not significantly different from wild type at $23.1 \pm 4.1 \mathrm{~cm}$ and $101.4 \pm 27.7$ seeds (unpaired $t$-tests; assessed at 12 weeks using three independent growth trials with at least 15 plants per trial; Figure 2). Germination rate was also the same at $90.4 \pm 10.9 \%, 90.0 \pm 13.1 \%$, and $91.25 \pm 11.3 \%$, for wild type, prOmiRBdIRI-1e, and prOmiRBdIRI-3c lines, respectively ( 8 independent growth trials using at least 10 seeds per trial).

\subsection{Antifreeze Activity and Freeze Resistance}

The ability to knockdown AFP expression was tested in "splat" assays to visualise IRI activity in crude extracts or apoplast samples (Figure 3, Figure S7). CA knockdown prOmiBdIRI-1e and prOmiRBdIRI-3c lines all showed reduced AFP activity compared to wild type CA plants and were similar to assays of NA wild type and NA transgenic lines. A dilution series used to estimate the difference in activity showed that at $0.01 \mathrm{mg} \mathrm{mL}^{-1}$, CA knockdown lines and NA wild type show larger ice crystals at the conclusion of the annealing period compared to samples from CA wild type and CA prOmiReGFP. Thus, CA appears to regulate the rice promoter to drive the miRNA to attenuate the expression of the AFP gene products, but it is possible that endogenous BdIRI 
438

439

440

promoters may have some very low levels of expression under NA conditions. As well, although the two lines both show attenuation of AFP activity, there appeared to be minor differences in expression, undoubtedly due to position effects, as has been shown in other transgene insertions (e.g. Simón-Mateo and Garcia, 2006; Duan et al., 2008; Liu et al., 2017).

TH was significantly increased to $0.05^{\circ} \mathrm{C}$ in wild type tissue extracts from CA plants compared to being virtually undetectable in NA samples (Table 1; Figure S5). Plant AFPs have TH levels that are typically low with ice-purified $B d$ AFPs previously reported at $0.08{ }^{\circ} \mathrm{C}$ (Bredow et al., 2016). CA transgenic prOmiRBdIRI-1e and prOmiRBdIRI-3c had TH activities that were significantly reduced (77\% and 74\%) compared to the levels shown by CA wild type. Strikingly, these low-temperature induced transgenic knockdowns showed levels of $\mathrm{TH}$ at $0.013{ }^{\circ} \mathrm{C}$ and 0.012 ${ }^{\circ} \mathrm{C}$, comparable to previously reported constitutive knockdowns expressing the same miRNA, at a $\mathrm{TH}$ range of $0.009-0.034{ }^{\circ} \mathrm{C}$ (Table 1 and Bredow et al., 2016), confirming the successful silencing of $B d$ AFP activity even when the miRNA was driven by the temporal rice promoter.

Brachypodium AFPs shape ice into hexagon crystals followed by a smooth, irregular, flowershaped burst (Figure S6). As would be expected, samples from CA wild type plants showed obvious ice shaping, but some minimal shaping still occurred in NA wild type and knockdown samples (Figure S7), consistent with the IRI results. Ice shaping in the CA knockdowns appeared to occur on the primary prism plane, favouring slight shaping along the $a$-axis before weakly bursting (Figure S7). In comparison, the CA wild type showed initial adsorption affinity for the $a$-axis primary prism plane quickly followed by adsorption and shaping on the $c$-axis basal plane, forming stunted hexagonal bipyramidal forms with strong bursts when the freezing point was exceeded. Rounded $B d$ AFP-mediated ice burst morphology presumably would help protect membranes by preventing the growth of large, sharp ice crystals as is seen in some freeze-avoiding organisms (Bar Dolev et al., 2016). Such membrane protection can be quantitatively assessed by electrolyte leakage assays. The CA transgenic leaves showed a significant increase $(\sim 30 \%)$ in electrolyte leakage $(p<0.05$, one-way ANOVA) at $-10^{\circ} \mathrm{C}$ when compared to CA wild type (Figure 4). At higher freezing temperatures of $6{ }^{\circ} \mathrm{C}$, electrolyte leakage was variably increased in the CA knockdown lines relative to CA wild type leaves (not shown). In all experiments, leaves from plants maintained at $4{ }^{\circ} \mathrm{C}$ showed relatively little electrolyte leakage independent of the genotype, as expected.

When entire plants were frozen to $-8{ }^{\circ} \mathrm{C}, 47 \%$ of CA wild type survived, significantly more ( $p$ $<0.01$, unpaired $t$-test) compared to $20 \%$ of CA prOmiRBdIRI-1e plants and none of the CA prOmiRBdIRI-3c plants (Figure 5). In addition, none of the NA plants survived, independent of genotype. To further assess ice propagation and freezing patterns of the temporal knockdown compared to wild type plants, infrared thermography was used as encouraged by previous observations of ice nucleation and propagation in various species (Wisniewski et al., 1997; Lutze et al., 1998; Ball et al., 2002; Sekozawa et al., 2004; Wisniewski et al., 2015). Images of CA and NA freezing leaf tissue from wild type compared to knockdown lines as the temperature was reduced to $10{ }^{\circ} \mathrm{C}$ were distinct, suggesting that AFPs can slow the propagation of ice through leaf tissues, and were consistent with the electrolyte leakage assays (Figure 6). Thermographs further supported the conclusion that ice propagation at subzero temperatures was more rapid in leaves from AFP knockdown lines. Temperature readings collected concurrently with thermographs on leaves show CA wild type leaves are $1-2{ }^{\circ} \mathrm{C}$ warmer than the NA wild type and CA knockdowns, consistent with the known $\sim 2{ }^{\circ} \mathrm{C}$ freezing point depression of INPs mediated by BdAFPs with a divergence around -2 ${ }^{\circ} \mathrm{C}$ where INPs nucleate ice (Figure S8). 
Ice nucleation under natural conditions is invariably initiated by INA+ bacteria, including strains of $P$. syringae, but as noted, Brachypodium AFPs can attenuate INPs in vitro (Bredow et al., 2018). To measure the impact of $B d$ AFPs on pathogen infection, wild type and a knockdown line were exposed to $P$. syringae pv. syringae B728A, a pathovar with INPs (Feil et al., 2005). Leaves from NA wild type and CA knockdown lines treated with the bacterial culture and placed at $-3{ }^{\circ} \mathrm{C}$, a temperature below which this pathovar nucleates ice (Figure S9A), displayed disease-like symptoms including water soaking and cell death, while infected plants that were not subjected to freezing temperatures displayed non-freeze-associated infection symptoms $12 \mathrm{~h}$ post infection (Figure S9BS9E). In contrast, CA wild type leaves displayed little evidence of tissue damage (Figure S10). After 24-48 h post infection, leaves from plants known to have little or no AFP activity were shrivelled and dry compared to CA wild type leaves, likely due to cell lysis associated with ice nucleation and growth. These same leaves showed more disease symptoms and cell death one week after pathovar exposure compared to CA wild type controls. Although it is not surprising that treated CA wild type leaves showed symptoms of disease considering the bacterial titre used, they appeared to have greater resistance to dehydration compared to the leaves with low AFP activity. This qualitative assay suggests that AFPs could ameliorate the impact of pathogens with INA activity.

\section{Discussion}

\subsection{Heterologous Promoter-Driven Expression}

The decision around the selection of an appropriate promoter to drive transcription in transgenic organisms is frequently challenging. Regulatory sequences including enhancers associated with the promoter can dictate tissue and developmental expression and thus transcription of a coding sequence of interest can be finely tuned particularly in model organisms where sequence libraries of promoters and enhancers are available, such as the rich resources available for Drosophila geneticists. For grasses, genetic resources are scarcer and in the absence of annotated promoter sequences, constitutive promoters such as CaMV 35S are used. However, their employment can risk inappropriate expression, epigenetic silencing, and even suboptimal growth (Estrada-Melo et al., 2015; Rajeevkumar et al., 2015; Amack and Antunes, 2020). Thus, the short stature, low germination rate, and the near-sterility in transgenic plants generated using the CamV 35S promoter ligated to a sequence for a miRNA that targets the translation of BdIRI gene products was unfortunate but not unexpected. Previous empty vector control plants did not present pleiotropic effects (Bredow et al., 2016), and thus these detrimental characteristics could be associated with the constitutive presence of the miRNA, and either due to the attenuation of AFPs and/or LRRs perhaps needed during stem elongation and seed formation, or simply the expression of these unregulated miRNAs recruiting polymerases or polysomes and thus interfering with proper development.

As an alternative to constitutive promoters, synthetic promoters consisting of core promoters and combinations of enhancer sequences for regulatory elements, sometimes from heterologous species, can be constructed with uncertain outcomes (Mohan et al., 2017; Ali and Kim, 2019). Again, as another somewhat risky strategy, an entire promoter sequence from evolutionarily related plants with documented expression profiles similar to the target genes in a second species can be selected and may allow for a greater degree of control including spatio-temporal expression (Dutt et al., 2014). Likewise, heterologous promoters may be free from endogenous signalling that could lead to undesired effects if using a native host promoter (Napoli et al., 1990). For the BdAFPs that are known to be produced within two days of CA, the cold-regulated promoter from $O$. sativa was an attractive choice even though we were unaware if it had been previously used heterologously. In rice it is induced at $4{ }^{\circ} \mathrm{C}$, the CA temperature of Brachypodium, and the directed rice transcripts increase 
continuously for $48 \mathrm{~h}$ (Li et al., 2017). Here we show that the use of this rice promoter indeed functions in Brachypodium and there were no obvious detrimental phenotypes associated with its use, either in the transgenics bearing the miRNA sequence or in the eGFP expression plants (Figure 2).

Non-coding RNAs including miRNAs are part of a complex signalling network having roles

\subsection{AFP Knockdowns and Freezing Vulnerability} in developmental regulation and stress response with multiple targets for single miRNAs (Chen, 2009; Peter, 2010; Wu et al., 2010; Budak and Akpinar, 2015; Liu et al., 2017; Waititu et al., 2020). Complementary sequence targets include gene transcripts that can be hydrolysed, translationally inhibited, or can localise to the nucleus and target promoter sequences (Jones-Rhoades et al., 2006; Li et al., 2013; Yang et al., 2019). Their frequent roles in the regulation of stress responses are of interest with regards to genes induced after CA. The Brachypodium Poaceae lineage diverged from rice $\sim 50$ million years ago, with high homology and synteny remaining between Brachypodium and rice genomes (Bossolini et al., 2007; Huo et al., 2009; Kumar et al., 2009; IBI, 2010). The identification of Brachypodium miRNA predicted target sequences in both the BdIRI and the $O S M Y B 1 R 35$ promoters suggests an evolutionarily conserved low temperature response that encouraged the prospect that the rice promoter would not only be recognized but could be appropriately regulated by any of the identified Brachypodium miRNAs with target sites in the rice sequence (Table S1; Table S2).

As noted, the transgenic lines appeared to have no developmental defects and as such the observation that they displayed similar freeze susceptibility as those lines bearing a constitutive promoter driving the miRNA translational interference of AFPs convincingly demonstrates that AFPs do contribute to freeze tolerance. In wild type Brachypodium, a short two-day CA period is both necessary and sufficient to prepare the plants for survival to subzero temperatures and is coincident with the appearance of AFPs. These AFPs depress the freezing point of solutions only marginally, but more importantly depress pathogen-mediated INA, shape ice, restrict ice crystal growth as measured by IRI, reduce electrolyte leakage, and ultimately allow whole plant survival even after freezing. Especially striking was the killing of all plants in one line and a second line showing less than half the survival rate compared to wild type controls at $-8{ }^{\circ} \mathrm{C}$. All these AFP-related properties were effectively knocked down by the temperature-regulated response of the rice promoter to direct miRNA expression (Figures 3, 4 and 5).

It has been assumed that AFPs are associated with the plasma membrane since reports of a fish AFP bound to model lipid bilayers changed the phase transition and prompted the researchers to recommend the use of fish AFPs to confer low temperature survival to plants, which ultimately did not meet with success (Tomczak et al., 2002; Kenward et al., 1993; Kenward et al., 1999). In plants, the localization of AFPs in the apoplast would suggest limited access to plasma membranes in any case. Indeed, mass spectrophotometry of CA Brachypodium plasma membrane proteins did not reveal any BdAFPs (Juurakko et al. 2021b). Nonetheless, AFPs do protect plasma membranes from damage, presumably from uncontrolled ice growth initiated by nucleators in the apoplast with its low solute concentration, and across cell walls into the cells.

The apoplast location of the AFPs allowed their assay in the absence of many other contaminating proteins and yielded clear evidence of the knockdown of IRI activity in the CA transgenic lines with the appearance of large ice crystals after the annealing period (Figure 3 ). Activity in lysates was consistent but not as visually clear, although individual ice crystals were substantially larger in dilute samples from the knockdowns compared to controls. As judged by 
571

572

573

574

575

576

577

578

579

580

581

582

583

584

585

586

587

588

589

590

591

592

593

594

595

596

597

598

599

600

601

602

603

604

605

606

607

608

609

610

611

612

613

614

615

western blot analysis of samples from transgenic plants expressing eGFP, the cold-induced rice promoter was not strong in Brachypodium. Thus, it is surprising that after a two day induction period, the heterologous sequence-directed miRNA lines showed AFP activities similar to the earlierreported constitutively expressed miRNA lines with respect to IRI, electrolyte leakage, whole plant freezing survival, and TH (Figures 3, 4, 5, and S5 vs. Bredow et al., 2016). This suggests that the single miRNA designed to complement the multiple BdIRI transcripts was very effective notwithstanding the heterologous promoter and the miRNA's less than perfect "match", underscoring the effectiveness of knockdown by translational attenuation (Bredow et al., 2016).

Structural ice barriers and thermal decoupling controlled by plant architecture and morphology, well known in angiosperms between stems and flowers, may not be as easily applied to grasses (Kuprian et al., 2014; Bertel et al., 2021). No evidence of either were observed in freezing Brachypodium leaves analysed by infrared thermography. However, the presence of AFPs in CA wild type tissue was coincident with the obvious retarded advancement of freeze fronts, a lag that was on average $1-2{ }^{\circ} \mathrm{C}$ different in leaves with AFPs than without, and coincidentally similar to the $\sim 2{ }^{\circ} \mathrm{C}$ attenuation of ice nucleation activity by BdAFPs (Bredow et al., 2018, Figure S9). There was no inhibition of ice front development observed in NA wild type and CA AFP knockdown lines suggesting that AFPs can delay ice propagation in leaf tissue, consistent with IRI, electrolyte leakage, and whole plant freezing assays. Since mass spectrophotometric analysis has shown that $B d$ AFPs encoded by BdIRI3 and 4 were detected in leaf tissue (Bredow et al., 2016), at least these two isoforms are associated with leaf ice growth protection. Adsorption of these AFPs to forming ice crystals will keep crystals small and less damaging to adjacent membranes thus limiting leaf damage.

Similar to the attenuation of freezing by AFPs in leaves was the retardation of damage subsequent to infection as seen after exposure of wounded leaves to P. syringae pv. syringae B728A. This pathovar can nucleate ice at high subzero temperatures presumably as a means to destroy tissues and access nutrients (Feil et al., 2005). It is known that BdAFPs inhibit the activity of the P. syringae INP in vitro (Bredow et al. 2018) and we speculate that a physical interaction of the INP and BdAFPs is sufficient to impact bacterial fitness, such that the progress of infection was slowed down. This effect was seen as early as 12-48 $\mathrm{h}$ after the pathogen challenge, and with the knockdowns showing more cellular death one week post-infection. Thus, it would be of interest in the future to more fully explore the relationship between AFP activity and the infectivity of pathogens bearing INPs.

The cause of the developmental effects seen in constitutive knockdowns of BdIRI (Bredow et al., 2016) is unknown. One potential reason may be the miRNA-mediated loss of the LRR isoforms that are hydrolysed in the apoplast from the primary translation products. In general, LRRs function in protein-protein interactions and have roles in growth and development in many organisms, normally in association with various other proteins. As well as LRR-receptor kinases that recognize apoplastic peptides to initiate immune signalling (Fischer et al., 2016) or regulate plant growth and development (He et al., 2018), LRR-extension proteins (LRRX) are bound to cell walls and function in growth as well as pollen tube formation (Zhao et al., 2018). However, to our knowledge, the characterisation and function of plant LRR domains in the absence of adjoining ligands has not been reported and any impact of their knockdown during development is unknown. Instead, the defective phenotypes may have simply arisen due to the constitutive-driven strong miRNA expression nonspecifically interfering with gene regulation and polysome loading during critical developmental stages, or alternatively, the possibility that AFPs have an as yet undiscovered role in Brachypodium development.

\subsection{Conclusions and Future Prospects}


Taken together, we have shown that substituting a constitutive promoter for a heterologous

618 temporally regulated promoter to direct the expression of a miRNA that targets the BdIRI transcripts substantially reduced AFP activity but with no apparent developmental defects. These results highlight the potential of Brachypodium AFPs as candidates for the development of freeze-tolerant horticultural crops, if not food crops where there may be public resistance to genetically modified plants. Additional biotechnological and research applications extend outside agriculture and range from additives to prevent ice recrystallization in stored cells and tissues, processed foods, and pharmaceuticals, particularly where infrastructure for flash freezing and extremely low temperature storage is not viable or otherwise unavailable. This research also opens the way to explore in planta the effects of AFPs on freezing tolerance and pathogen susceptibility as well as the means by which BdIRIs may be regulated through cis-regulatory elements and endogenous miRNAs.

\section{Conflict of Interest}

628 The authors declare that the research was conducted in the absence of any commercial or financial 629 relationships that could be construed as a potential conflict of interest.

\section{Author Contributions}

631 CLJ conducted all experiments, analysed all data, and produced all figures. CLJ wrote the initial draft

632 of the manuscript and all authors contributed to manuscript revision. MB assisted in the design of

633 some early experiments and conducted preliminary infection experiments. VKW and GCD co-

634 supervised and secured funding.

\section{$635 \quad 7 \quad$ Funding}

636 Research in the VKW and GCD laboratories is supported through Discovery Grants from the Natural 637 Sciences and Engineering Research Council of Canada.

\section{Acknowledgments}

639 We acknowledge Dr. Barabara Vanderbeld for her previous published work on the miRNA construct, 640 Dr. Heather Tomalty and Robert Eves (Dr. Peter L. Davies' lab) for use of the nanoliter osmometer, 641 Jeff Boudreau (Dr. Ian Chin-Sang's lab) for assistance with the western blots, Kristy Moniz for 642 assisting with plant care, and Ryan Monday for helping to harvest tens of thousands of seeds, 643 phenotyping hundreds of plants, and assisting with plant care.

\section{Supplementary Material}

645 Supplementary Tables S1 and S2 are available online at: (link here).

\section{$646 \quad 10 \quad$ References}

647 Ali, S., \& Kim, W. C. (2019). A fruitful decade using synthetic promoters in the improvement of 648 transgenic plants. Frontiers in plant science, 10, 1433.

649 Amack, S. C., \& Antunes, M. S. (2020). CaMV35S promoter-A plant biology and biotechnology 650 workhorse in the era of synthetic biology. Current Plant Biology, 100179. 
Antikainen, M., \& Griffith, M. (1997). Antifreeze protein accumulation in freezing-tolerant cereals.

Armenteros, J. J. A., Sønderby, C. K., Sønderby, S. K., Nielsen, H., \& Winther, O. (2017). DeepLoc: 654 prediction of protein subcellular localization using deep learning. Bioinformatics, 33(21), 3387-3395.

655 Armenteros, J. J. A., Tsirigos, K. D., Sønderby, C. K., Petersen, T. N., Winther, O., Brunak, S., ... \& 656 Nielsen, H. (2019). SignalP 5.0 improves signal peptide predictions using deep neural networks. 657 Nature biotechnology, 37(4), 420-423.

Ashworth, E. N., \& Kieft, T. L. (1995). Ice nucleation activity associated with plants and fungi. In:

660 Baev, V., Milev, I., Naydenov, M., Apostolova, E., Minkov, G., Minkov, I., \& Yahubyan, G. (2011). 661 Implementation of a de novo genome-wide computational approach for updating Brachypodium 662 miRNAs. Genomics, 97(5), 282-293.

663 Ball, M. C., Wolfe, J., Canny, M., Hofmann, M., Nicotra, A. B., \& Hughes, D. (2002). Space and 664 time dependence of temperature and freezing in evergreen leaves. Functional Plant Biology, 29(11), $665 \quad 1259-1272$.

666 Bar Dolev, M., Braslavsky, I., \& Davies, P. L. (2016). Ice-binding proteins and their function. 667 Annual review of biochemistry, 85, 515-542.

668 Ben-Amar, A., Oueslati, S., \& Mliki, A. (2017). Universal direct PCR amplification system: a time669 and cost-effective tool for high-throughput applications. 3 Biotech, 7(4), 246.

670 Bertel, C., Hacker, J., \& Neuner, G. (2021). Protective Role of Ice Barriers: How Reproductive 671 Organs of Early Flowering and Mountain Plants Escape Frost Injuries. Plants, 10(5), 1031.

672 Bertolini, E., Verelst, W., Horner, D. S., Gianfranceschi, L., Piccolo, V., Inzé, D., ... \& Mica, E. 673 (2013). Addressing the role of microRNAs in reprogramming leaf growth during drought stress in 674 Brachypodium distachyon. Molecular plant, 6(2), 423-443.

675 Blum, M., Chang, H. Y., Chuguransky, S., Grego, T., Kandasaamy, S., Mitchell, A., ... \& Finn, R. D. 676 (2021). The InterPro protein families and domains database: 20 years on. Nucleic Acids Research, 677 49(D1), D344-D354.

678 Bossolini, E., Wicker, T., Knobel, P. A., \& Keller, B. (2007). Comparison of orthologous loci from 679 small grass genomes Brachypodium and rice: implications for wheat genomics and grass genome 680 annotation. The Plant Journal, 49(4), 704-717.

681 Bredow, M., \& Walker, V. K. (2017). Ice-binding proteins in plants. Frontiers in Plant Science, 8, 6822153.

683 Bredow, M., Tomalty, H. E., Smith, L., \& Walker, V. K. (2018). Ice and anti-nucleating activities of 684 an ice-binding protein from the annual grass, Brachypodium distachyon. Plant, cell \& environment, 685 41(5), 983-992. 
Bredow, M., Tomalty, H. E., \& Walker, V. K. (2017). Identification of plant ice-binding proteins through assessment of ice-recrystallization inhibition and isolation using ice-affinity purification. JoVE (Journal of Visualized Experiments), (123), e55302.

689 Bredow, M., Vanderbeld, B., \& Walker, V. K. (2016). Knockdown of ice-binding proteins in 690 Brachypodium distachyon demonstrates their role in freeze protection. PloS one, 11(12), e0167941.

691 Budak, H., \& Akpinar, B. A. (2015). Plant miRNAs: biogenesis, organization and origins. Functional 692 \& integrative genomics, 15(5), 523-531.

693 Chen, C. (2015). Determining the leaf emissivity of three crops by infrared thermometry. Sensors, 694 15(5), 11387-11401.

695 Colton-Gagnon, K., Ali-Benali, M. A., Mayer, B. F., Dionne, R., Bertrand, A., Do Carmo, S., \& Charron, 696 J. B. (2014). Comparative analysis of the cold acclimation and freezing tolerance capacities of seven 697 diploid Brachypodium distachyon accessions. Annals of Botany, 113(4), 681-693.

698 Dai, X., Zhuang, Z., \& Zhao, P. X. (2018). psRNATarget: a plant small RNA target analysis server 699 (2017 release). Nucleic Acids Research, 46(W1), W49-W54.

700 Duan, C. G., Wang, C. H., Fang, R. X., \& Guo, H. S. (2008). Artificial microRNAs highly accessible 701 to targets confer efficient virus resistance in plants. Journal of Virology, 82(22), 11084-11095.

Duman, J. G. (2001). Antifreeze and ice nucleator proteins in terrestrial arthropods. Annual Review of 703 Physiology, 63(1), 327-357.

704

Dutt, M., Dhekney, S. A., Soriano, L., Kandel, R., \& Grosser, J. W. (2014). Temporal and spatial control of gene expression in horticultural crops. Horticulture research, 1(1), 1-17.

706 biosynthesis gene using a stress-inducible promoter enhances drought resistance in petunia.

Feil, H., Feil, W. S., Chain, P., Larimer, F., DiBartolo, G., Copeland, A., ... \& Lindow, S. E. (2005). pv. tomato DC3000. Proceedings of the National Academy of Sciences, 102(31), 11064-11069.

712 Fischer, I., Diévart, A., Droc, G., Dufayard, J. F., \& Chantret, N. (2016). Evolutionary dynamics of 713 the leucine-rich repeat receptor-like kinase (LRR-RLK) subfamily in angiosperms. Plant physiology, 714 170(3), 1595-1610.

715 Fujii, H., Chiou, T. J., Lin, S. I., Aung, K., \& Zhu, J. K. (2005). A miRNA involved in phosphate716 starvation response in Arabidopsis. Current Biology, 15(22), 2038-2043.

717 Fursova, O., Pogorelko, G., \& Zabotina, O. A. (2012). An efficient method for transient gene 718 expression in monocots applied to modify the Brachypodium distachyon cell wall. Annals of Botany, 719 110(1), 47-56.

720 Gasteiger, E., Hoogland, C., Gattiker, A., Wilkins, M. R., Appel, R. D., \& Bairoch, A. (2005).

721 Protein identification and analysis tools on the ExPASy server. In The proteomics protocols

722 handbook (pp. 571-607). Humana press. 
723

724

725

726

727

728

729

730

731

732

733

734

735

736

737

738

739

740

741

742

743

744

745

746

747

748

749

750

751

752

753

754

755

756
Griffith, M., Ala, P., Yang, D. S., Hon, W. C., \& Moffatt, B. A. (1992). Antifreeze protein produced endogenously in winter rye leaves. Plant Physiology, 100(2), 593-596.

Griffiths-Jones, S. (2004). The microRNA registry. Nucleic acids research, 32(suppl_1), D109D111.

Griffiths-Jones, S., Grocock, R. J., Van Dongen, S., Bateman, A., \& Enright, A. J. (2006). miRBase: microRNA sequences, targets and gene nomenclature. Nucleic acids research, 34(suppl_1), D140D144.

Griffiths-Jones, S., Saini, H. K., Van Dongen, S., \& Enright, A. J. (2007). miRBase: tools for microRNA genomics. Nucleic acids research, 36(suppl_1), D154-D158.

He, Y., Zhou, J., Shan, L., \& Meng, X. (2018). Plant cell surface receptor-mediated signaling-a common theme amid diversity. Journal of Cell Science, 131(2), jcs209353.

Hon, W. C., Griffith, M., Chong, P., \& Yang, D. S. (1994). Extraction and isolation of antifreeze proteins from winter rye (Secale cereale L.) leaves. Plant Physiology, 104(3), 971-980.

Huo, N., Vogel, J. P., Lazo, G. R., You, F. M., Ma, Y., McMahon, S., .. \& Gu, Y. Q. (2009). Structural characterization of Brachypodium genome and its syntenic relationship with rice and wheat. Plant molecular biology, 70(1-2), 47-61.

International Brachypodium Initiative (IBI). (2010). Genome sequencing and analysis of the model grass Brachypodium distachyon. Nature, 463, 763-768.

Jones-Rhoades, M. W., \& Bartel, D. P. (2004). Computational identification of plant microRNAs and their targets, including a stress-induced miRNA. Molecular Cell, 14(6), 787-799.

Jones-Rhoades, M. W., Bartel, D. P., \& Bartel, B. (2006). MicroRNAs and their regulatory roles in plants. Annual Review of Plant Biology, 57, 19-53.

Juurakko, C. L., diCenzo, G. C., \& Walker, V. K. (2021a). Cold acclimation and prospects for coldresilient crops. Plant Stress, 2, 100028.

Juurakko, C. L., Bredow, M., Nakayama, T., Imai, H., Kawamura, Y., diCenzo, G. C., ... \& Walker, V. K. (2021b). The Brachypodium distachyon cold-acclimated plasma membrane proteome is primed for stress resistance. G3, 11(9), jkab198.

Kelley, L. A., Mezulis, S., Yates, C. M., Wass, M. N., \& Sternberg, M. J. (2015). The Phyre2 web portal for protein modeling, prediction and analysis. Nature protocols, 10(6), 845-858.

Kenward, K. D., Altschuler, M., Hildebrand, D., \& Davies, P. L. (1993). Accumulation of type I fish antifreeze protein in transgenic tobacco is cold-specific. Plant molecular biology, 23(2), 377-385.

Kenward, K. D., Brandle, J., McPherson, J., \& Davies, P. L. (1999). Type II fish antifreeze protein accumulation in transgenic tobacco does not confer frost resistance. Transgenic research, 8(2), 105117. 
757

758

759

760

761

762

763

764

765

766

767

768

769

770

771

772

773

774

775

776

777

778

779

780

781

782

783

784

785

786

787

788

789

790

791

792

Kim, H. J., Lee, J. H., Hur, Y. B., Lee, C. W., Park, S. H., \& Koo, B. W. (2017). Marine antifreeze proteins: structure, function, and application to cryopreservation as a potential cryoprotectant. Marine drugs, 15(2), 27.

Kozomara, A., \& Griffiths-Jones, S. (2010). miRBase: integrating microRNA annotation and deepsequencing data. Nucleic acids research, 39(suppl_1), D152-D157.

Kozomara, A., \& Griffiths-Jones, S. (2014). miRBase: annotating high confidence microRNAs using deep sequencing data. Nucleic Acids Research, 42(D1), D68-D73.

Kozomara, Ana, Maria Birgaoanu, and Sam Griffiths-Jones (2019). "miRBase: from microRNA sequences to function." Nucleic Acids Research 47(D1), D155-D162.

Kreyling, J. (2019). The ecological importance of winter in temperate, boreal, and arctic ecosystems in times of climate change. In Progress in Botany Vol. 81 (pp. 377-399). Springer, Cham.

Kumar, S., Mohan, A., Balyan, H. S., \& Gupta, P. K. (2009). Orthology between genomes of Brachypodium, wheat and rice. BMC research notes, 2(1), 1-9.

Kuprian, E., Briceño, V. F., Wagner, J., \& Neuner, G. (2014). Ice barriers promote supercooling and prevent frost injury in reproductive buds, flowers and fruits of alpine dwarf shrubs throughout the summer. Environmental and experimental botany, 106, 4-12.

Lescot, M., Déhais, P., Thijs, G., Marchal, K., Moreau, Y., Van de Peer, Y., ... \& Rombauts, S. (2002). PlantCARE, a database of plant cis-acting regulatory elements and a portal to tools for in silico analysis of promoter sequences. Nucleic acids research, 30(1), 325-327.

Li, J., Qin, R., Xu, R., Li, H., Yang, Y., Li, L., Wei, P., \& Yang, J. (2017). Isolation and identification of five cold-inducible promoters from Oryza sativa. Planta, 247(1), 99-111.

Li, S., Liu, L., Zhuang, X., Yu, Y., Liu, X., Cui, X., ... \& Chen, X. (2013). MicroRNAs inhibit the translation of target mRNAs on the endoplasmic reticulum in Arabidopsis. Cell, 153(3), 562-574.

Lindow, S. E., Arny, D. C., and Upper, C. D. (1982). Bacterial ice nucleation: a factor in frost injury to plants. Plant Physiology, 70, 1084-1089.

Liu, S. R., Zhou, J. J., Hu, C. G., Wei, C. L., \& Zhang, J. Z. (2017). MicroRNA-mediated gene silencing in plant defense and viral counter-defense. Frontiers in Microbiology, 8, 1801.

López, A., Molina-Aiz, F. D., Valera, D. L., \& Peña, A. (2012). Determining the emissivity of the leaves of nine horticultural crops by means of infrared thermography. Scientia Horticulturae, 137 , 49-58.

Lutze, J. L., Roden, J. S., Holly, C. J., Wolfe, J., Egerton, J. J. G., \& Ball, M. C. (1998). Elevated atmospheric [CO2] promotes frost damage in evergreen tree seedlings. Plant, Cell \& Environment, 21(6), 631-635.

Lv, D. W., Zhen, S., Zhu, G. R., Bian, Y. W., Chen, G. X., Han, C. X., ... \& Yan, Y. M. (2016). High-throughput sequencing reveals $\mathrm{H} 2 \mathrm{O} 2$ stress-associated microRNAs and a potential regulatory network in Brachypodium distachyon seedlings. Frontiers in plant science, 7, 1567. 
793 Mallory, A. C., \& Vaucheret, H. (2006). Functions of microRNAs and related small RNAs in plants.

794 Nature genetics, 38(6), S31-S36.

795 Marentes, E., Griffith, M., Mlynarz, A., \& Brush, R. A. (1993). Proteins accumulate in the apoplast 796 of winter rye leaves during cold acclimation. Physiologia Plantarum, 87(4), 499-507.

797 Mayer, B. F., Bertrand, A., \& Charron, J. B. (2020). Treatment analogous to seasonal change 798 demonstrates the integration of cold responses in Brachypodium distachyon. Plant Physiology, 182(2), $799 \quad 1022-1038$.

800 Melo-Abreu, J. P. D., Villalobos, F. J., \& Mateos, L. (2016). Frost protection. In Principles of 801 Agronomy for Sustainable Agriculture (pp. 443-457). Springer, Cham.

802 Middleton, A. J., Vanderbeld, B., Bredow, M., Tomalty, H., Davies, P. L., \& Walker, V. K. (2014). 803 Isolation and characterization of ice-binding proteins from higher plants. In Plant Cold Acclimation 804 (pp. 255-277). Humana Press, New York, NY.

805 Mohan, C., Jayanarayanan, A. N., \& Narayanan, S. (2017). Construction of a novel synthetic root806 specific promoter and its characterization in transgenic tobacco plants. 3 Biotech, 7(4), 1-9.

807 Napoli, C., Lemieux, C., \& Jorgensen, R. (1990). Introduction of a chimeric chalcone synthase gene 808 into petunia results in reversible co-suppression of homologous genes in trans. The plant cell, 2(4), 809 279-289.

810 NOAA National Centers for Environmental Information (NCEI) U.S. Billion-Dollar Weather and 811 Climate Disasters (2021). https://www.ncdc.noaa.gov/billions/.

812 Pearce, R. S., \& Fuller, M. P. (2001). Freezing of barley (Hordeum) studied by infrared video 813 thermography. Plant Physiology, 125, 227-240.

814 Peter, M. E. (2010). Targeting of mRNAs by multiple miRNAs: the next step. Oncogene, 29(15), 815 2161-2164.

816 Pogorelko, G., Fursova, O., Lin, M., Pyle, E., Jass, J., \& Zabotina, O. A. (2011). Post-synthetic

817 modification of plant cell walls by expression of microbial hydrolases in the apoplast. Plant

818 molecular biology, 77(4), 433-445.

819 Qin, Y., He, Y., Wu, B., Ma, S., \& Zhang, X. (2017). Regulating top albedo and bottom emissivity of 820 concrete roof tiles for reducing building heat gains. Energy and Buildings, 156, 218-224.

821 Rajeevkumar, S., Anunanthini, P., \& Ramalingam, S. (2015). Epigenetic silencing in transgenic 822 plants. Frontiers in Plant Science, 6, 693.

823 Ramsay, J. A. (1964). The rectal complex of the mealworm Tenebrio molitor, L.(Coleoptera, 824 Tenebrionidae). Philosophical Transactions of the Royal Society of London. Series B, Biological 825 Sciences, 248(748), 279-314.

826 Sekozawa, Y., Sugaya, S., \& Gemma, H. (2004). Observations of ice nucleation and propagation in 827 flowers of Japanese pear (Pyrus pyrifolia Nakai) using infrared video thermography. Journal of the 828 Japanese Society for Horticultural Science, 73(1), 1-6. 
829 Sievers, F., Wilm, A., Dineen, D., Gibson, T. J., Karplus, K., Li, W., ... \& Thompson, J. D. (2011).

830 Fast, scalable generation of high-quality protein multiple sequence alignments using Clustal Omega.

831 Molecular systems biology, 7(1), 539.

832 Simón-Mateo, C., \& García, J. A. (2006). MicroRNA-guided processing impairs Plum pox virus

833 replication, but the virus readily evolves to escape this silencing mechanism. Journal of Virology,

834 80(5), 2429-2436.

835 Sinha, T., \& Cherkauer, K. A. (2010). Impacts of future climate change on soil frost in the

836 midwestern United States. Journal of Geophysical Research: Atmospheres, 115(D8).

837 Smith, A. B., \& Katz, R. W. (2013). US billion-dollar weather and climate disasters: Data sources, 838 trends, accuracy and biases. Natural Hazards, 67(2), 387-410.

839 Smith, A., Lott, N., Houston, T., Shein, K., Crouch, J., \& Enloe, J. (2021). US Billion-Dollar

840 Weather \& Climate Disasters 1980-2021. NOAA National Centers for Environmental Information:

841 Asheville, NC, USA, 15.

842 Tomalty, H. E., \& Walker, V. K. (2014). Perturbation of bacterial ice nucleation activity by a grass

843 antifreeze protein. Biochemical and biophysical research communications, 452(3), 636-641.

844 Tomczak, M. M., Hincha, D. K., Estrada, S. D., Wolkers, W. F., Crowe, L. M., Feeney, R. E., ... \&

845 Crowe, J. H. (2002). A mechanism for stabilization of membranes at low temperatures by an

846 antifreeze protein. Biophysical journal, 82(2), 874-881.

847 Waititu, J. K., Zhang, C., Liu, J., \& Wang, H. (2020). Plant Non-Coding RNAs: origin, biogenesis, 848 mode of action and their roles in abiotic stress. International Journal of Molecular Sciences, 21(21), 8498401.

850 Witney, G. W., \& Arpaia, M. L. (1991). Tree recovery after the December 1990 freeze. California 851 Avocado Society 1991 Yearbook, 75, 63-70.

852 Wisniewski, M., \& Fuller, M. (1999). Ice nucleation and deep supercooling in plants: new insights

853 using infrared thermography. In Cold-Adapted Organisms (pp. 105-118). Springer, Berlin,

854 Heidelberg.

855 Wisniewski, M., Lindow, S. E., \& Ashworth, E. N. (1997). Observations of ice nucleation and 856 propagation in plants using infrared video thermography. Plant Physiology, 113(2), 327-334.

857 Wisniewski, M., Neuner, G., \& Gusta, L. V. (2015). The use of high-resolution infrared

858 thermography (HRIT) for the study of ice nucleation and ice propagation in plants. JoVE (Journal of

859 Visualized Experiments), (99), e52703.

860 Wu, S., Huang, S., Ding, J., Zhao, Y., Liang, L., Liu, T., ... \& He, X. (2010). Multiple microRNAs

861 modulate p21Cip1/Waf1 expression by directly targeting its 3' untranslated region. Oncogene,

862 29(15), 2302-2308.

863 Yang, G., Li, Y., Wu, B., Zhang, K., Gao, L., \& Zheng, C. (2019). MicroRNAs transcriptionally 864 regulate promoter activity in Arabidopsis thaliana. Journal of integrative plant biology, 61(11),

$865 \quad 1128-1133$. 
866 Zhang, B. (2015). MicroRNA: a new target for improving plant tolerance to abiotic stress. Journal of 867 experimental botany, 66(7), 1749-1761.

868 Zhang, J., Xu, Y., Huan, Q., \& Chong, K. (2009). Deep sequencing of Brachypodium small RNAs at 869 the global genome level identifies microRNAs involved in cold stress response. BMC genomics, $870 \quad 10(1), 1-16$.

871 Zhao, C., Zayed, O., Yu, Z., Jiang, W., Zhu, P., Hsu, C. C., ... \& Zhu, J. K. (2018). Leucine-rich 872 repeat extensin proteins regulate plant salt tolerance in Arabidopsis. Proceedings of the National 873 Academy of Sciences, 115(51), 13123-13128. 
874 Table 1. Thermal hysteresis $(\mathrm{TH})$ readings done using crude protein extracts from leaf tissue lysates on cold-acclimated (CA) and non-acclimated (NA) Bd21 wild type and temporal cold-induced antifreeze protein knockdown lines prOmiRBdIRI-1e and prOmiRBdIRI-3c. Samples were tested at $40 \mathrm{mg} \mathrm{mL}^{-1}$ of total protein concentrated from crude cell extracts. Readings were captured using a

878 nanoliter osmometer. Assays were performed in triplicate and values shown are the average of three 879 replicates with standard deviation.

\begin{tabular}{ll}
\hline Sample & TH $\left({ }^{\circ} \mathbf{C}\right)$ \\
\hline Buffer & 0 \\
Wild Type, Bd21 (CA) & $0.050 \pm 0.019$ \\
Wild Type, Bd21 (NA) & $0.005 \pm 0.003$ \\
prOmiRBdIRI-1e (CA) & $0.013 \pm 0.002$ \\
prOmiRBdIRI-1e (NA) & $0.003 \pm 0.003$ \\
prOmiRBdIRI-3c (CA) & $0.012 \pm 0.011$ \\
prOmiRBdIRI-3c (NA) & $0.002 \pm 0.002$ \\
\hline
\end{tabular}




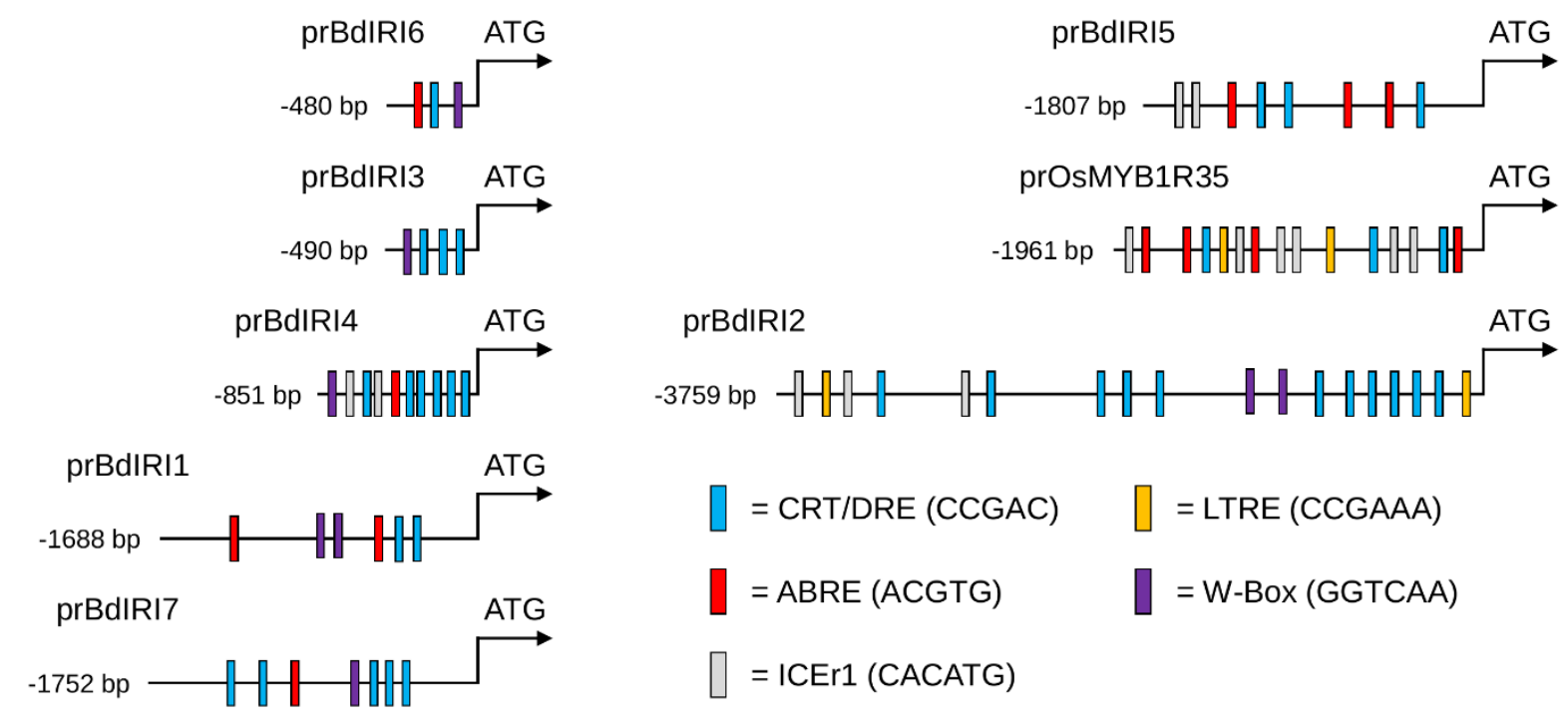

882 Figure 1. Illustrations of putative promoter regions 5' of the ATG start codon in the Brachypodium 883 distachyon AFP genes BdIRII-7 and the known cold-regulated promoter sequence of the rice, Oryza 884 sativa, gene OsMYB1R35. The analysis extended until the stop codon of the nearest upstream gene. 885 Cis-regulatory elements are annotated with strand positions shown relative to the sequence encoding 886 the ATG start. Coloured boxes correspond to canonical cold response related and drought resistant 887 element motifs (CRT/DRE; blue), the stress hormone, abscisic acid-responsive elements (ABREs; red), 888 the cold response pathway inducer of C-repeat binding factor expression 1 (ICEr1; grey), low889 temperature response elements (LTREs; yellow), and the WRKY stress transcription factors 890 recognition $\mathrm{W}$-box motifs (purple). 

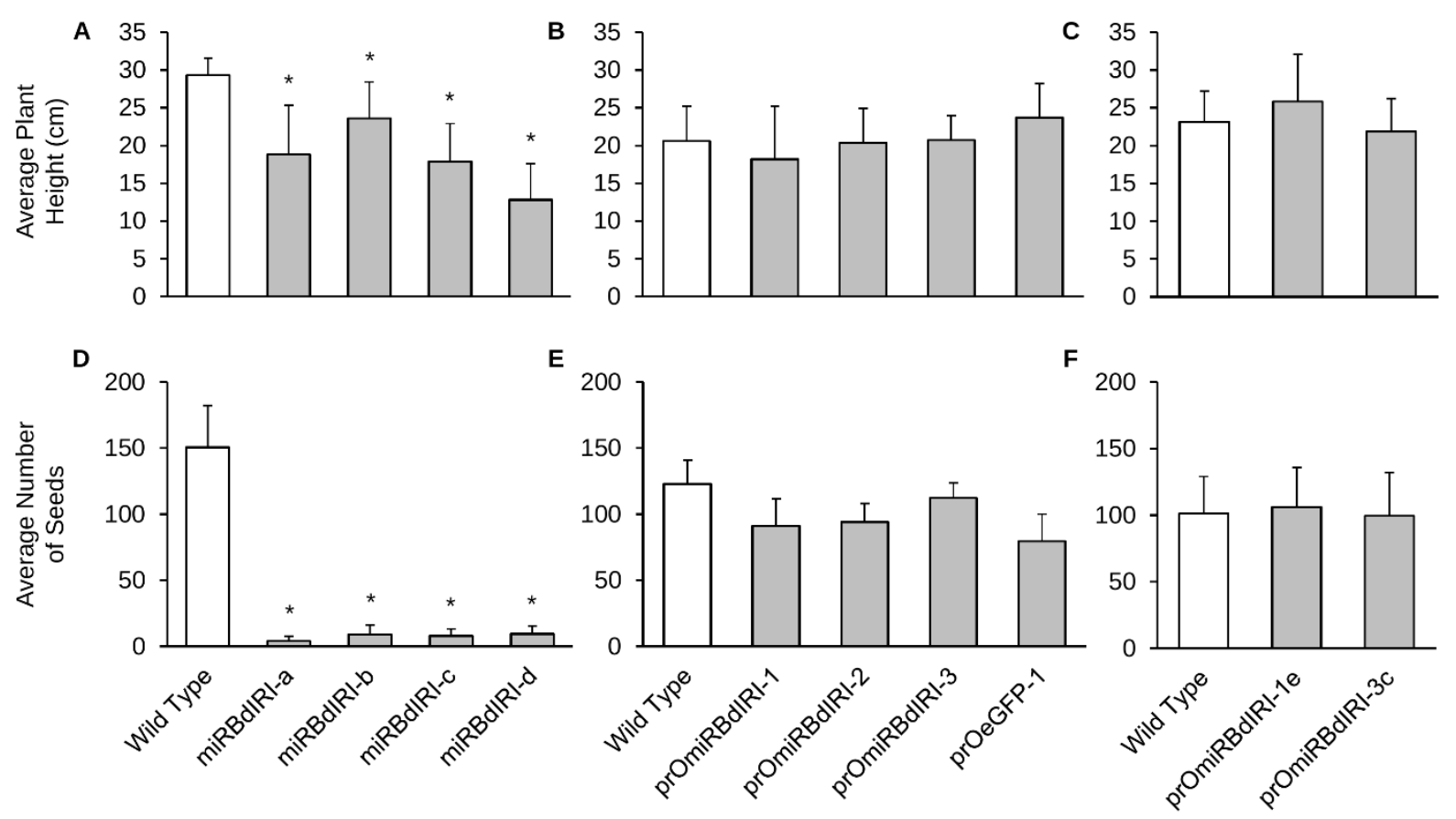

892 Figure 2. The average height and number of seeds per plant, in Brachypodium $\mathrm{Bd} 21$ wild type plants 893 and plants from transgenic lines. (A,D) Homozygous transgenic lines employing the CaMV 35S promoter ligated to the miRNA sequence to generate constitutively expressed BdIRI knockdown plants (data taken from Bredow et al.,2016). (B,E) Heterozygous temporal knockdown lines employing the OsMYB1R35 promoter, and (C,F) homozygous temporal knockdown lines. The data in B, C, E, and F and was compiled at 12 weeks from three independent growth trials using at least 15 plants per trial for each knockdown line and wild type. Asterisks indicate significant differences compared to wild 899 type (unpaired $t$-test, $p<5 \times 10^{-9}$ ). 


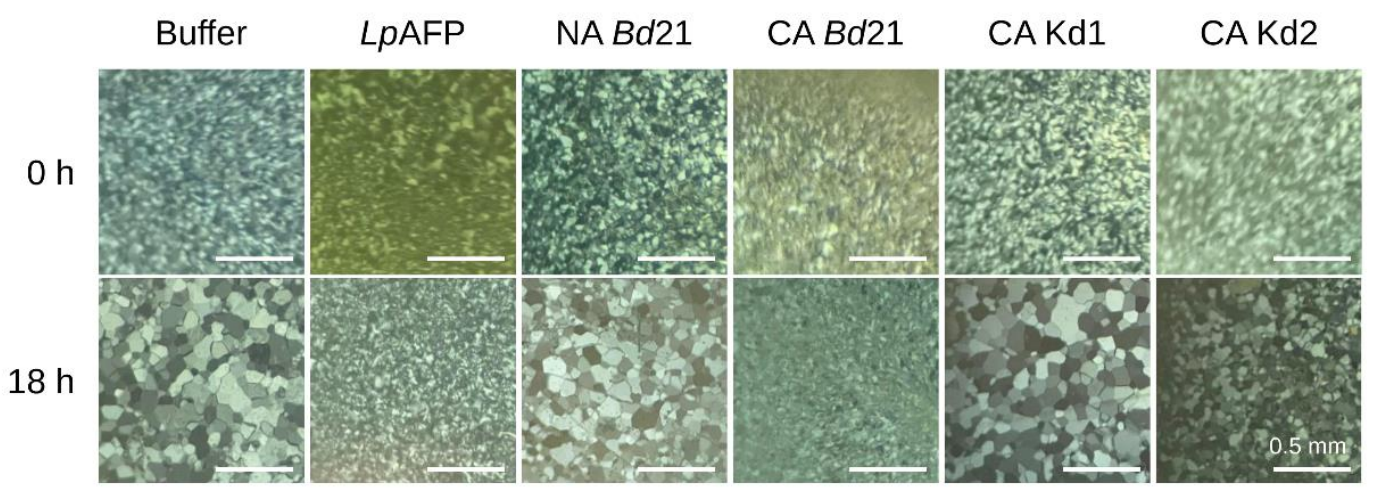

901 Figure 3. Ice recrystallization inhibition "splat" assays of apoplast extracts using non-acclimated (NA) 902 and cold-acclimated (CA) Brachypodium distachyon Bd21 wild type and temporal antifreeze protein 903 knockdown lines prOmiRBdIRI-1e (Kd1) and prOmiRBdIRI-3c (Kd2). Samples were annealed at -6 $904{ }^{\circ} \mathrm{C}$ for $18 \mathrm{~h}$ at a standardised concentration of $0.01 \mathrm{mg} \mathrm{mL}^{-1}$. Buffer and recombinant purified rye grass 905 (Lolium perenne AFP; LpAFP) controls are also shown. Assays were performed in triplicate with 906 similar results, and representative images are shown. Scale bars represent $0.5 \mathrm{~mm}$. Splat assays 907 performed using CA prOmiReGFP plants resulted in results similar to those obtained using wild type $908 \mathrm{Bd} 21$, indicating that plasmid presence and seed transformation did not affect AFP activity (not 909 shown). 


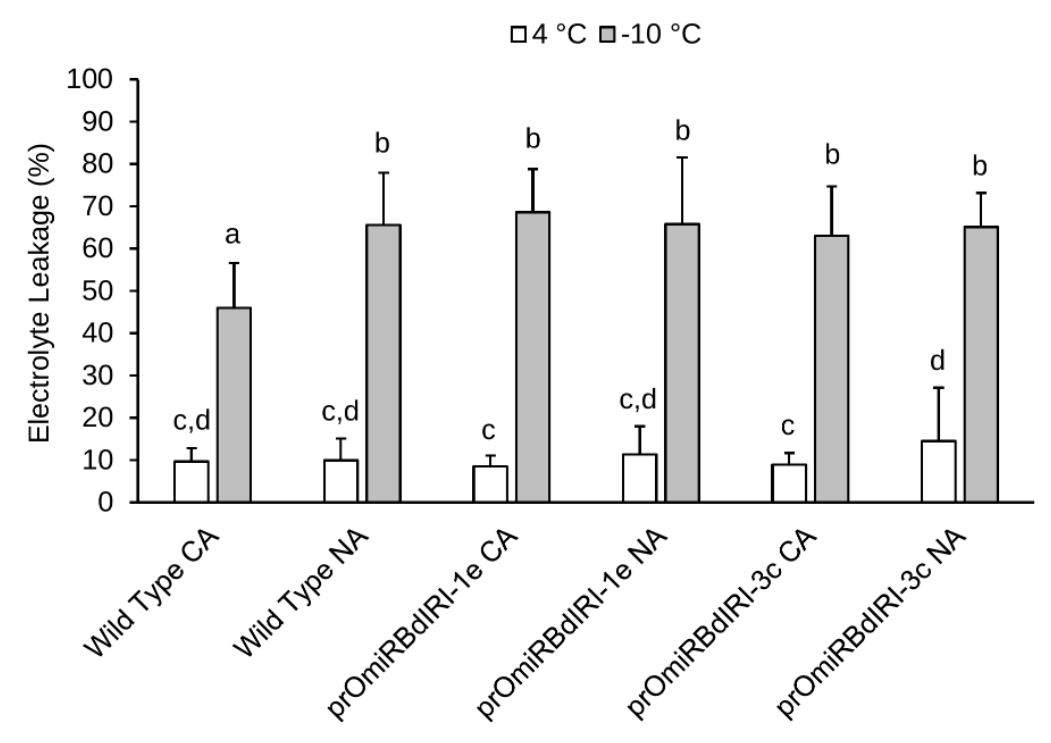

910

911 Figure 4. Electrolyte leakage assays performed on non-acclimated (NA) and cold-acclimated (CA)

912 wild type Brachypodium distachyon $\mathrm{Bd} 21$ and two homozygous temporal antifreeze protein 913 knockdown lines (prOmiRBdIRI-1e and prOmiRBdIRI-3c). Control leaves were maintained at $4{ }^{\circ} \mathrm{C}$ 914 (white bars) while experimental samples were incubated at $-10{ }^{\circ} \mathrm{C}$ (grey bars) as indicated. Electrolyte 915 leakage was measured as a percentage of electrolytes released after the freeze protocol as a ratio of the 916 total released electrolytes after autoclaving, based on the total leaf mass in the sample (see Methods). 917 Letters represent statistically significant groups following one-way ANOVA with post-hoc Tukey 918 multiple test correction $(p<0.05)$. Error bars represent the standard deviation of the mean and assays 919 were performed in triplicate $(n=10)$. 

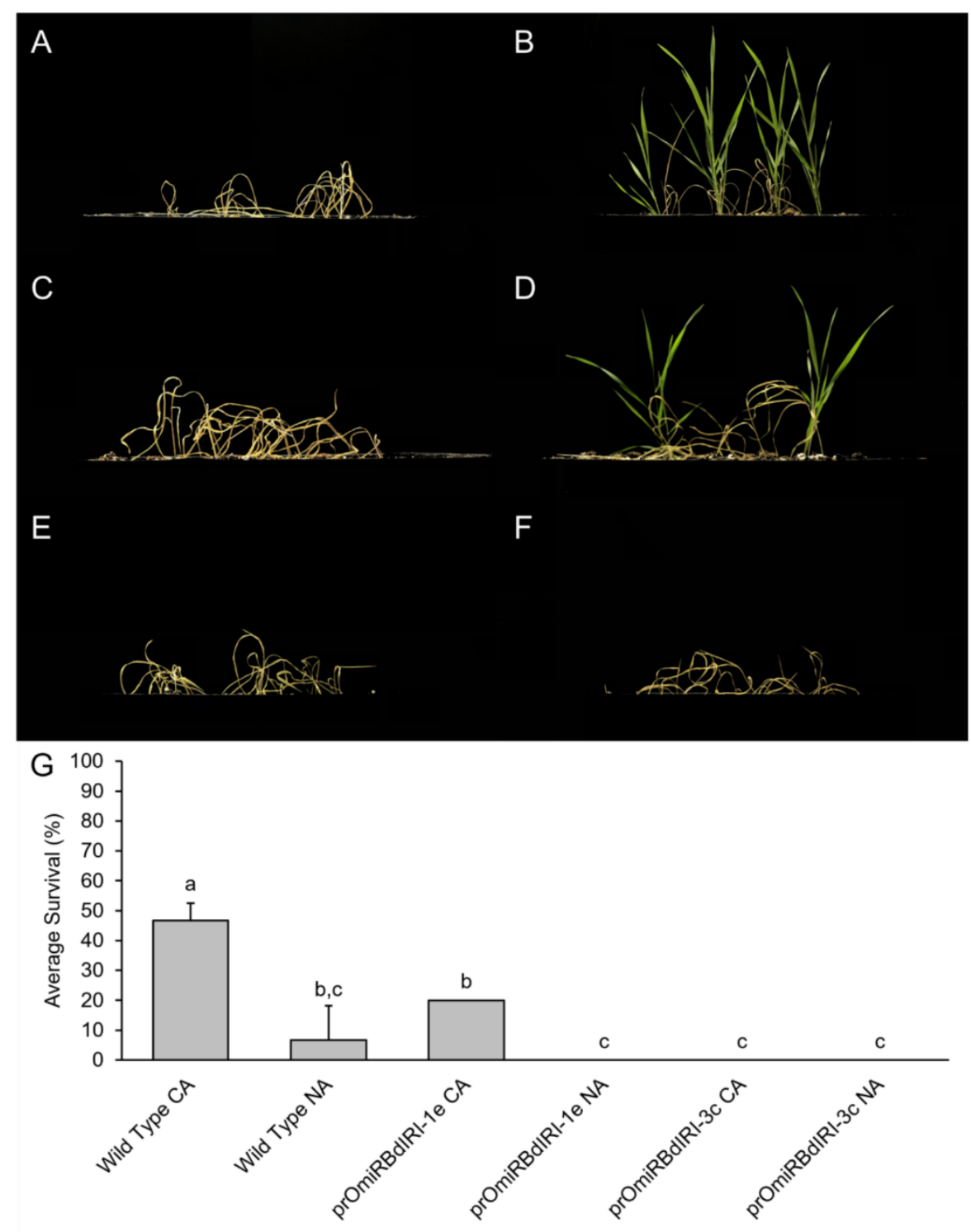

921 Figure 5. Whole plant freezing survival assay of non-acclimated (NA) and cold-acclimated (CA) wild 922 type Brachypodium distachyon Bd21 and two homozygous knockdown lines (prOmiRBdIRI-1e and 923 prOmiRBdIRI-3c). (A) NA Bd21 wild type. (B) CA Bd21 wild type. (C) NA prOmiRBdIRI-1e. (D) 924 CA prOmiRBdIRI-1e. (E) NA prOmiRBdIRI-3c. (F) CA prOmiRBdIRI-3c. (G) Values represent survival and are the average of three replicates, with error bars showing the standard deviation of the mean. Letters represent statistically significant groups following one-way ANOVA with post-hoc 927 Tukey multiple test correction $(p<0.01)$. Two-week-old plants were frozen at a rate of $1{ }^{\circ} \mathrm{C} \mathrm{h}^{-1}$ to -8 $928{ }^{\circ} \mathrm{C}$ in a temperature-controlled chamber in the dark following misting with sterile water to initiate 929 freezing. Plants were allowed to recover for two days at $4{ }^{\circ} \mathrm{C}$ with no light and returned to standard 930 conditions for 7 days before images $(A-F)$ were captured. Assays were performed in triplicate $(n=10)$. 

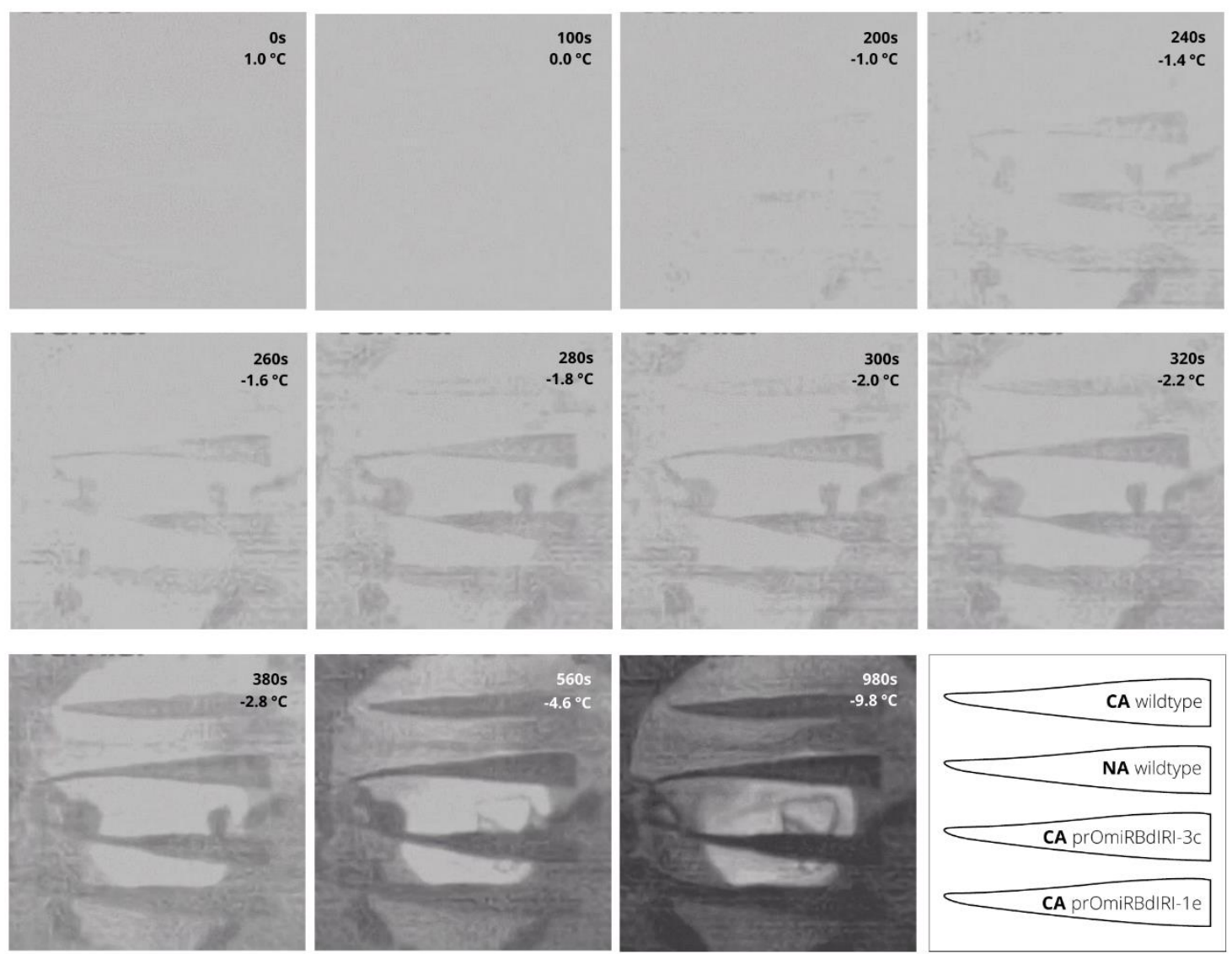

$1^{\circ} \mathrm{C}$

$-10^{\circ} \mathrm{C}$

932 Figure 6. Thermographs of excised leaf tissue of wild type cold-acclimated (CA), wild type non933 acclimated (NA), prOmiRBdIRI-1e CA, and prOmiRBdIRI-3c CA, from top to bottom, respectively. 934 Leaves were equilibrated at $1{ }^{\circ} \mathrm{C}$ and frozen to $-10{ }^{\circ} \mathrm{C}$. Ice propagation was nucleated by an ice chip 935 placed in $10 \mu \mathrm{L}$ of water on the excision wound. Time stamps and temperatures are indicated. A 936 diagram of the leaf samples is shown in bottom right with a temperature scale shown at the bottom. 
bioRxiv preprint doi: https://doi.org/10.1101/2022.02.15.480542; this version posted February 18, 2022. The copyright holder for this preprint (which was not certified by peer review) is the author/funder, who has granted bioRxiv a license to display the preprint in perpetuity. It is made available under aCC-BY 4.0 International license.

BdIRI1
BdIRI7
BdIRI5
BdIRI6
BdIRI4
BdIRI2
BdIRI3

BdIRI1 BdIRI 7

BdIRI 5

BdIRI 6

BdIRI 4

BdIRI2

BdIRI 3

BdIRI 1

BdIRI 7

BdIRI 5

BdIRI 6

BdIRI 4

BdIRI2

BdIRI 3

BdIRI 1

BdIRI 7

BdIRI 5

BdIRI 6

BdIRI 4

BdIRI2

BdIRI 3

BdIRI 1

BdIRI 7

BdIRI 5

BdIRI 6

BdIRI 4

BdIRI2

BdIRI 3

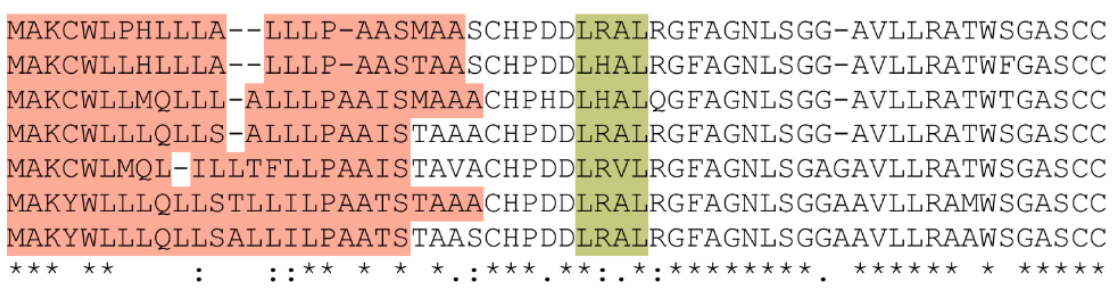

GWEGVGCDSA--SGRVTSLWLPGRGLTGPIQGAASLAGLVRLESLNLADNRLV--GTIPS GWEGVGCDGA--SGRVTTLWLPGRGLAGPIQGAASLAGLAQLESLNLANNRLV--GTIPS GWEGVGCNGGKSDGRVTTLLLPGRGLTGPI LGASSLAGLAQLESLNLAHNRLQVGSTFPL GWEGVGCDGD--AGRVTTLSLPERGLAGPIQGAAPLAGLRQLESLNLAHNRLQVGTTFPS SWEGVGCNGGA-SGRVMTLWLPGRGLAGPIQGAASLAGLARLESLNLANNRLQVGTTFPS GWEGVSCDGGA-SGRVTTLWLPGRGLVGPIQGVSSLAGLAQLESLNLANNRLQIGTTFPS GWEGVRCDDGA-SGRVTTLWLPGRGLVGPI HGASSLAGLAQLESLNLANNRLHVGTTFPS

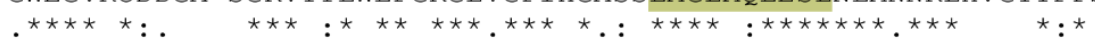

WIGELDRLCYLDLSHNASVYEVAKINPSQRSRGVTVSTNRKTLDEEPNTITGTNNHVRSG WIGELDRLCYLDLSHNASVDEVHKINPSQRSLGVAVS TNRRTLGGE PNTI TGTNNHVRSG WIGELGHLRYLDLSHNAS PLHVNSS------------NRRTLAEGQ PNT I SGTNNSVRSG WIAE FDRLCYLDLS HNVS PLHVKSS----------SRRTLATGQPNTI TGANNSVRSG WIGELDRLRYFDLS HNASSFHVKHG------------DRRTLADGQ PNTI TGTNNSVRSG WIGELDRLCYLDLS HNAS PLHVKR------------NRRNTS---------------WIGELDRLCYLDLSHNAS PLHVKR-------------NRRTLADGKPNT ITGTNNSVKSG

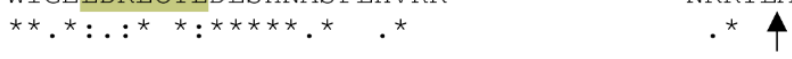

KDNALSGNDNTVISGDNNVVTGNHNKILSGSHNAVSGHMHVVSGTYHVVTGNNNAVTRSH KDNALSGSDNTVISGNNNVVT GNHNKVLSGSHNAVSGHMHVVSGTYHVVTGNNNAVTRSH SGNTVMGEDNIVISGDNNVVSGKQNT------------VTGSDNVVSGSNNSV----

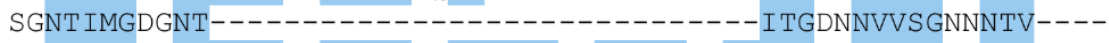
NGNTVSGGDNTVISGNNNVLSGNNNTI ISGSDNVVSGTNQVVSGTNHIVTGSNNTV---NGNT ISGNDNVVISGNNNVVSGNHNKVVSGSDNAVSGNMHVVSGTHHVVTGTNNTV----

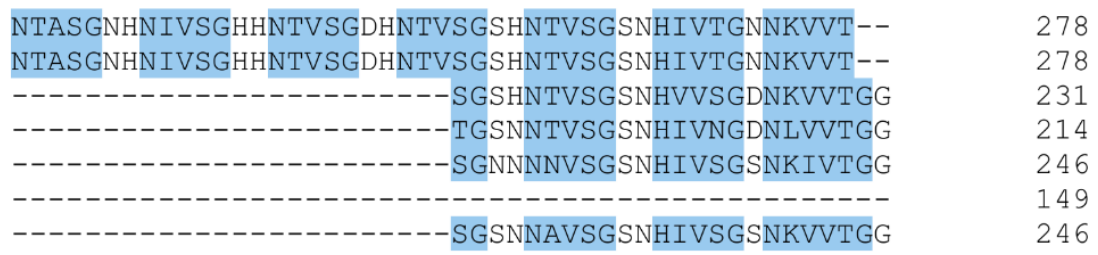

938 Figure S1. Alignments performed using Clustal Omega (https://www.ebi.ac.uk/Tools/msa/clustalo/) 939 of all 7 BdIRI amino acid sequences from the newest assembly (Brachypodium distachyon genome v3) 940 containing an annotated apoplast localization signal sequence in red, LRR motifs of LxxL where $x$ 941 represents a non-conserved residue in green, and AFP motifs of NxVxG/NxVxxG where $x$ represents 942 an outward-facing residue of the beta-barrel structure in blue, along with the putative asparagine 943 endopeptidase hydrolytic cleavage sites indicated by black arrows. Asterisks $(*)$ denote fully conserved 944 residues, colons (:) denote conservative substitutions, and periods (.) denote semi-conservative 945 substitutions. 


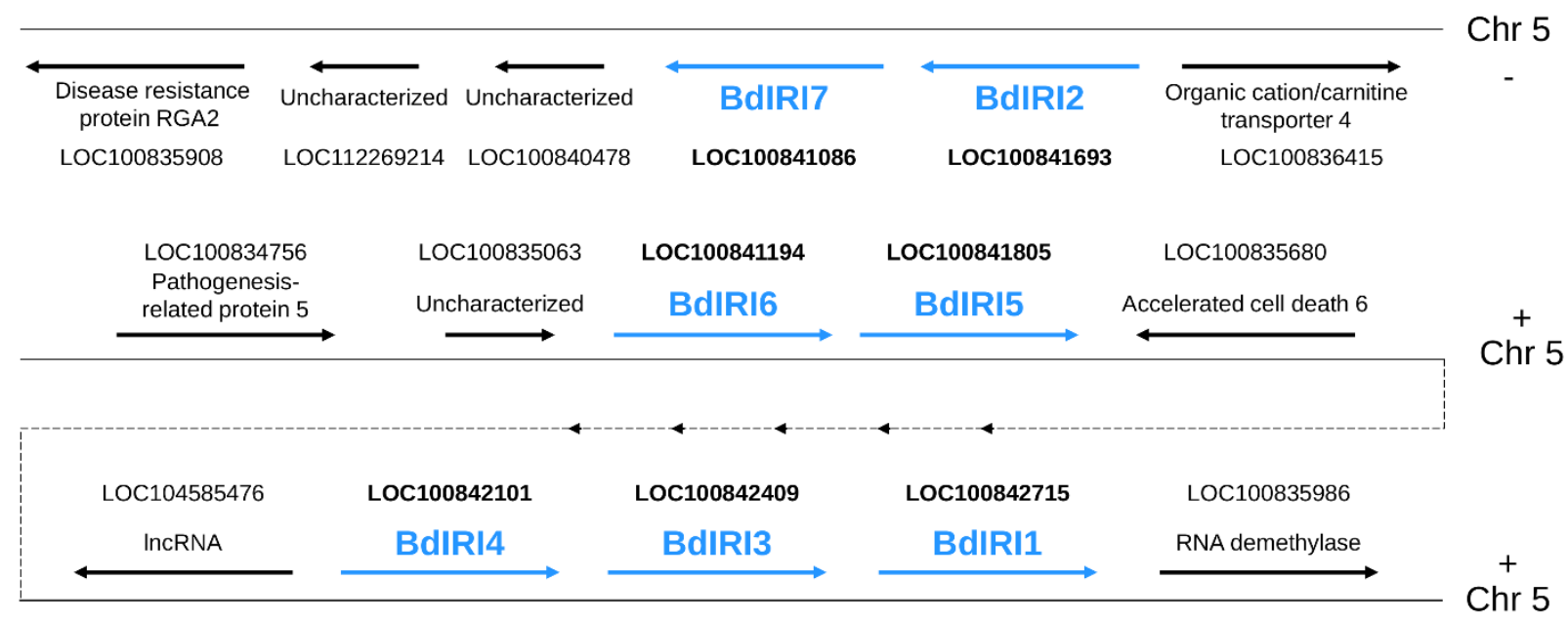

947 Figure S2. Illustration showing the three clusters and chromosomal positions of the 7 BdIRI genes on 948 chromosome five of Brachypodium (Brachypodium distachyon genome v3). BdIRI gene numbers are 949 labelled and strands (+/-) are labelled and are highlighted. Flanking and nearby genes are shown and $950 B d I R I$ genes are highlighted in bold. NCBI RefSeq loci are labelled. Dotted line indicates the direct 951 continuity of the chromosome at this position. 


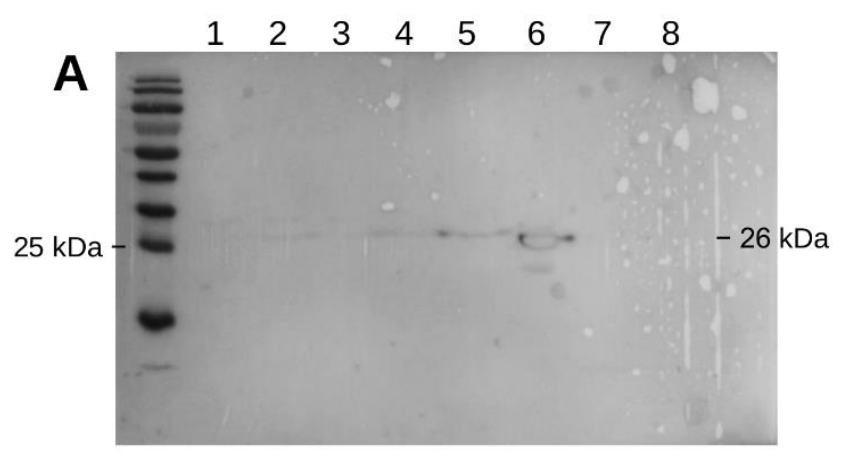

953 Figure S3. Representative western blot analysis for functional characterization of the rice promoter 954 prOsMYB1R35 system in Brachypodium distachyon using extracts from non-acclimated (NA) and

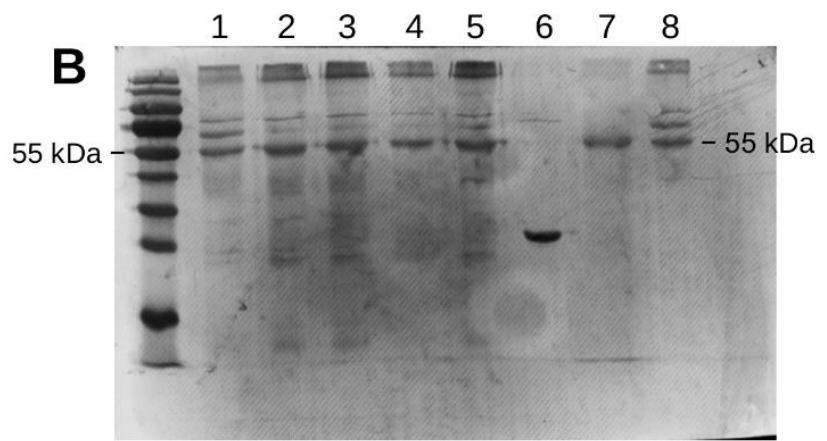
cold-acclimated (CA) Bd21 wild type and prOeGFP transgenic plants and visualised with antibodies for green fluorescent protein (GFP). (A) Lane 1 corresponds to NA prOeGFP, lanes 2-5 to CA prOeGFP from separate individual plants, lane 6 to to purified recombinant GFP used as a positive control, lane 7 to NA wild type, and lane 8 to CA wild type. (B) RuBisCO large chain (Rbcl) was used as a loading control with Coomassie Brilliant Blue (CBB) staining. Molecular weights of bands corresponding to eGFP, $26 \mathrm{kDa}$, and $\mathrm{Rbcl}, 55 \mathrm{kDa}$, are labelled. The western blots were performed in triplicate. Note: positive control recombinant eGFP was overloaded and burnt during imaging. 


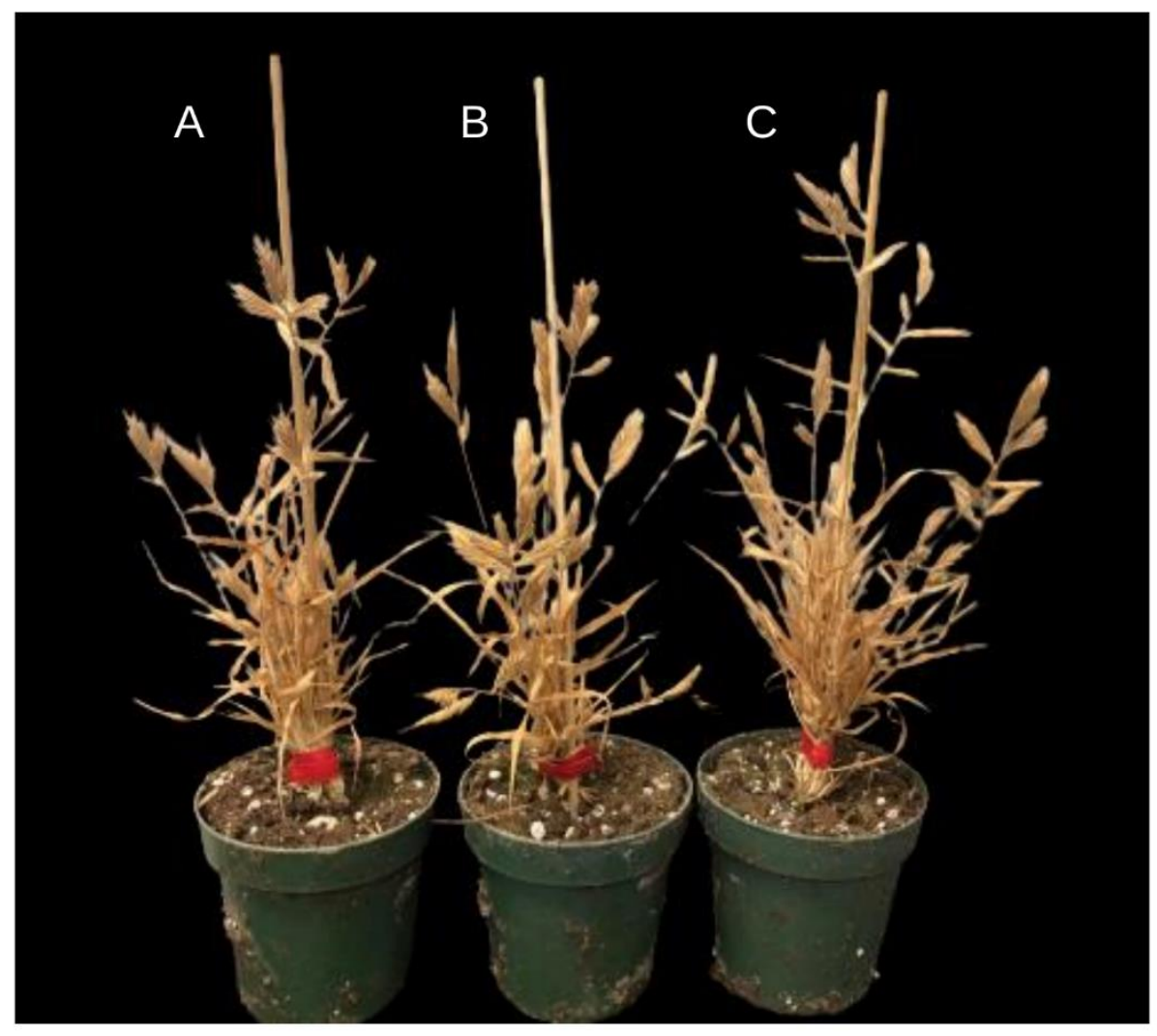

963 Figure S4. Phenotypes of Bd21 wild type Brachypodium distachyon (A) plants and two homozygous 964 knockdown lines, prOmiRBdIRI-1e (B) and prOmiRBdIRI-3c (C), bearing our temporal AFP 965 knockdown systems. Photos were taken at 12 weeks following the described standard growth 966 conditions with water and fertilizer withdrawn in the final week to allow senesced plants to dry out for 967 seed harvesting. 


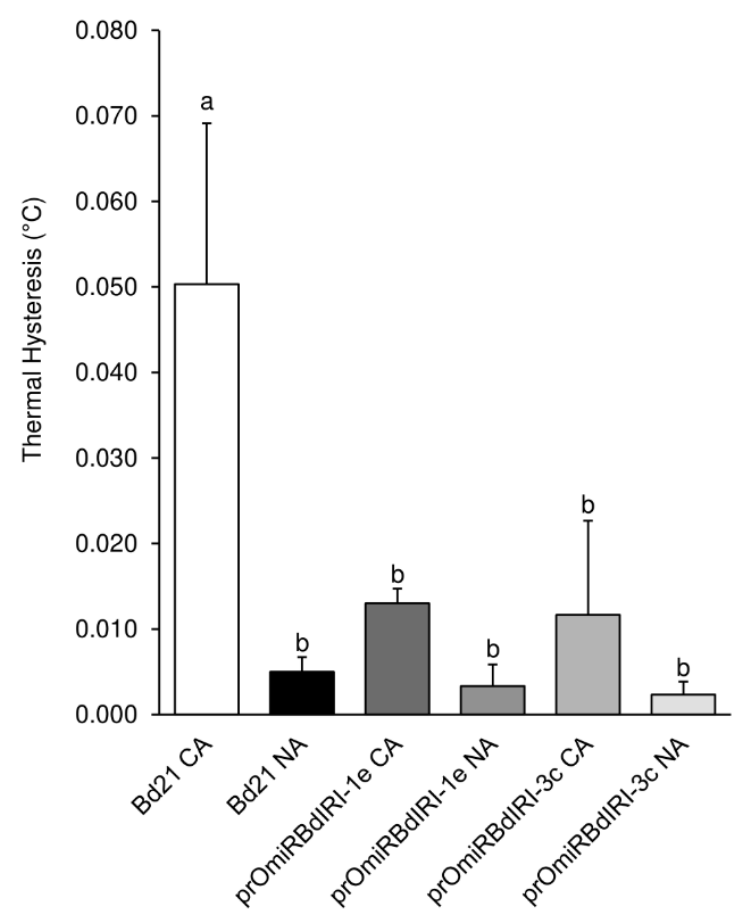

969 Figure S5. Thermal hysteresis (TH) readings done using crude protein extracts from leaf tissue lysates 970 on non-acclimated (NA) and cold-acclimated (CA) Bd21 wild type Brachypodium distachyon and two temporal AFP knockdown lines prOmiRBdIRI-1e and prOmiRBdIRI-3c. Samples were tested at 40 $\mathrm{mg} \mathrm{mL} \mathrm{m}^{-1}$ of total protein concentrated from crude cell extracts. Readings were captured using a nanoliter osmometer and performed in triplicate. Letters represent statistically significant groups following one-way ANOVA with post-hoc Tukey multiple test correction $(p<0.01)$. 

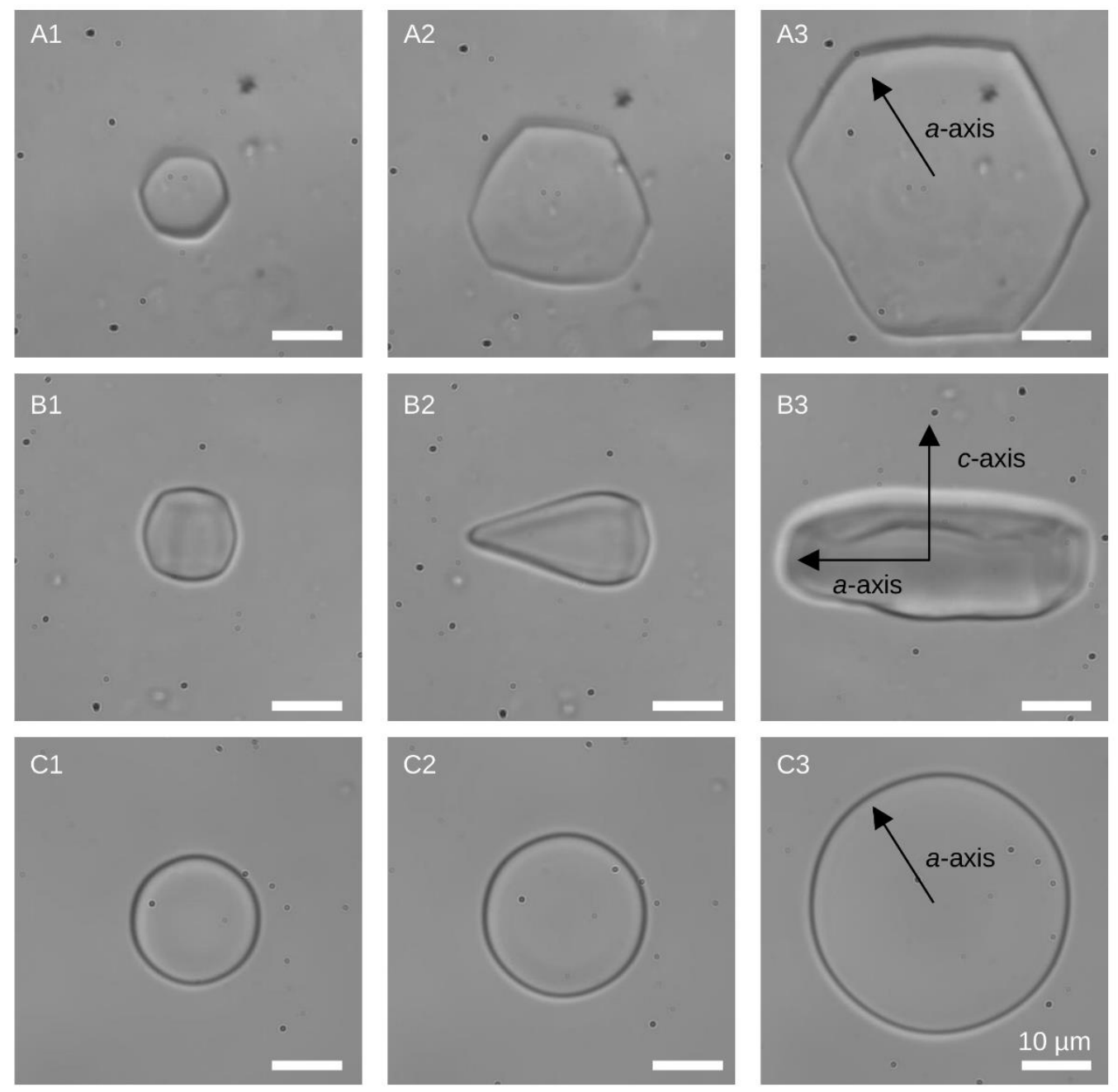

976 Figure S6. Ice crystal morphology and burst patterns in the presence of antifreeze proteins present in 977 crude lysates of wildtype cold-acclimated Brachypodium distachyon $\mathrm{Bd} 21$ leaf tissue viewed down on 978 the $a$-axis (A1-A3) and viewed horizontally towards the $a$-axis (B1-B3). Brachypodium AFPs have an 979 affinity for the prism and basal planes. Buffer solution showing unrestricted ice crystal growth in the 980 absence of AFPs with characteristic disk-shaped morphology visible, viewed down on the $a$-axis (C1981 C3). Micrographs were captured at 50x zoom on a nanoliter osmometer and performed in triplicate. 982 Scale bar is $10 \mu \mathrm{m}$. 

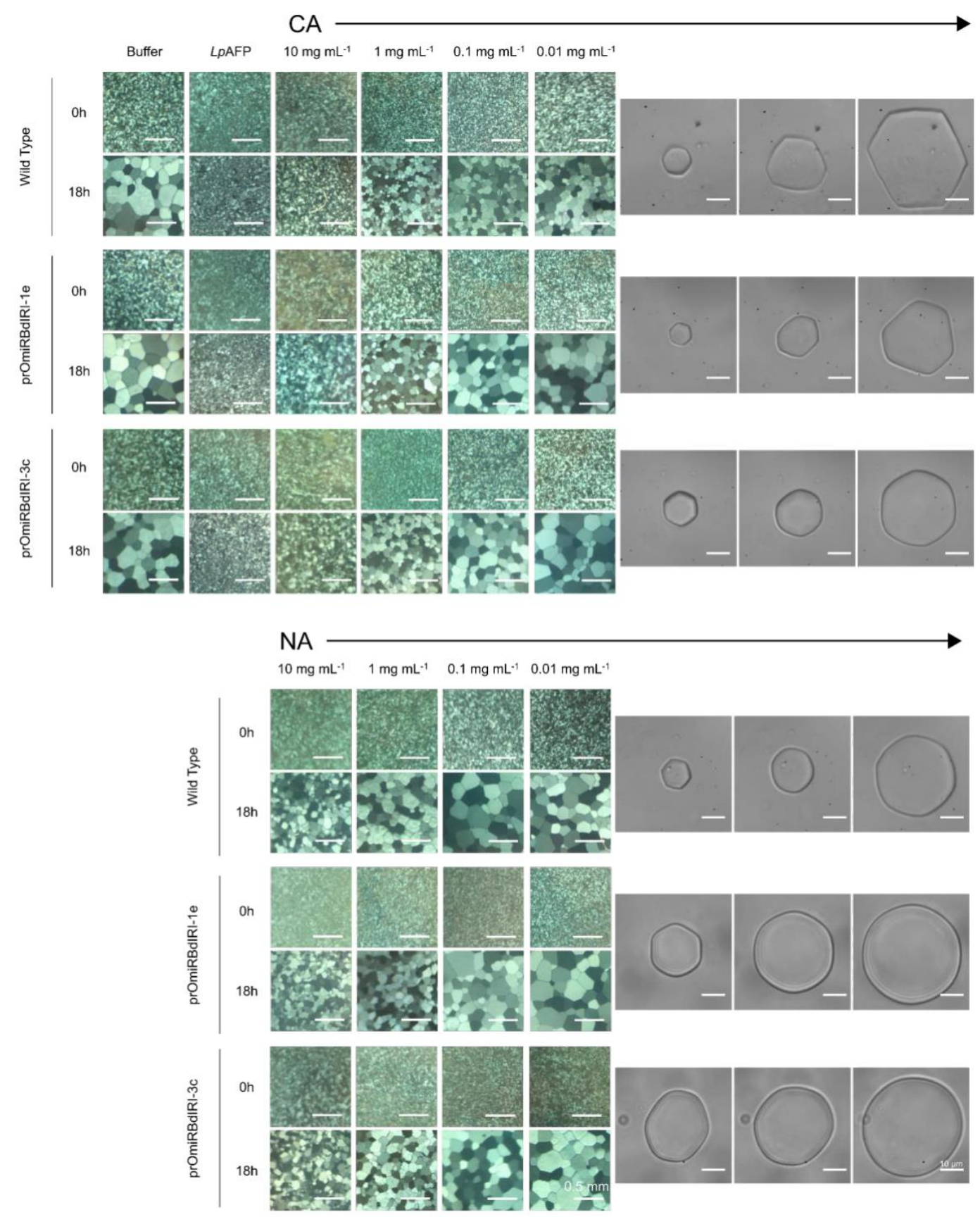

984 Figure S7. Ice recrystallization inhibition "splat" assays of cell protein extracts using leaf tissue from 985 non-acclimated (NA) and cold-acclimated (CA) Brachypodium distachyon Bd21 wild type and 986 temporal AFP knockdown lines prOmiRBdIRI-1e and prOmiRBdIRI-3c. Samples were annealed at $987 \quad 6{ }^{\circ} \mathrm{C}$ for $18 \mathrm{~h}$ at various concentrations. Buffer and LpAFP controls are shown. Scale bar for splat 988 assays represents $0.5 \mathrm{~mm}$. Ice crystal morphologies and burst patterns are shown alongside and were 989 tested at $40 \mathrm{mg} \mathrm{mL}^{-1}$ of total protein concentrated from crude cell extracts. Micrographs were captured 990 at 50x zoom on a nanoliter osmometer with scale bars representing $10 \mu \mathrm{M}$. All assays were performed 991 in triplicate with similar results and representative images are shown. 


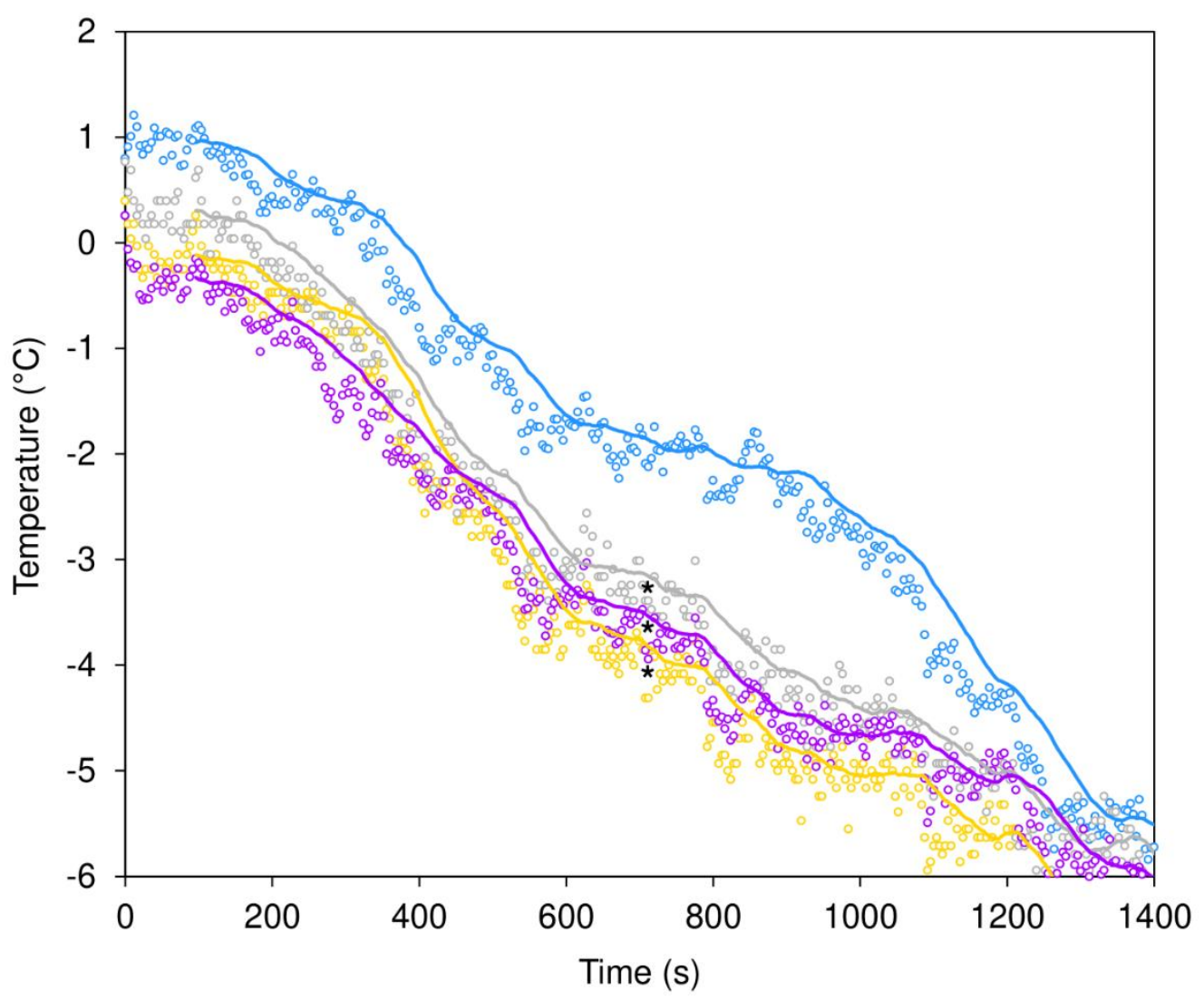

993 Figure S8. Infrared thermography assay performed on cold-acclimated (CA) wild type (blue), CA 994 prOmiRBdIRI-1e (grey), CA prOmiRBdIRI-3c (purple) knockdown lines, and non-acclimated (NA) 995 wild type (yellow). Data represent the recorded thermography data where each point represents the 996 temperature at a single frame captured at a frame rate of 1 frame every $4 \mathrm{sec}$. Lines shown represent 997 the moving averages using a period of 25. Points measured were $5 \mathrm{~mm}$ from the wounded end of the 998 leaf where the tissue was excised from plants. Leaves were equilibrated at $1{ }^{\circ} \mathrm{C}$ for 30 min and frozen 999 to $-10{ }^{\circ} \mathrm{C}$ at a rate of $0.01{ }^{\circ} \mathrm{C} \mathrm{sec}{ }^{-1}$. Experiments were performed in triplicate. CA prOmiRBdIRI-1e, 1000 CA prOmiRBdIRI-3c knockdown lines, and NA wild type were significantly different from CA wild 1001 type indicated by stars (one tailed $t$-test, unpaired). 

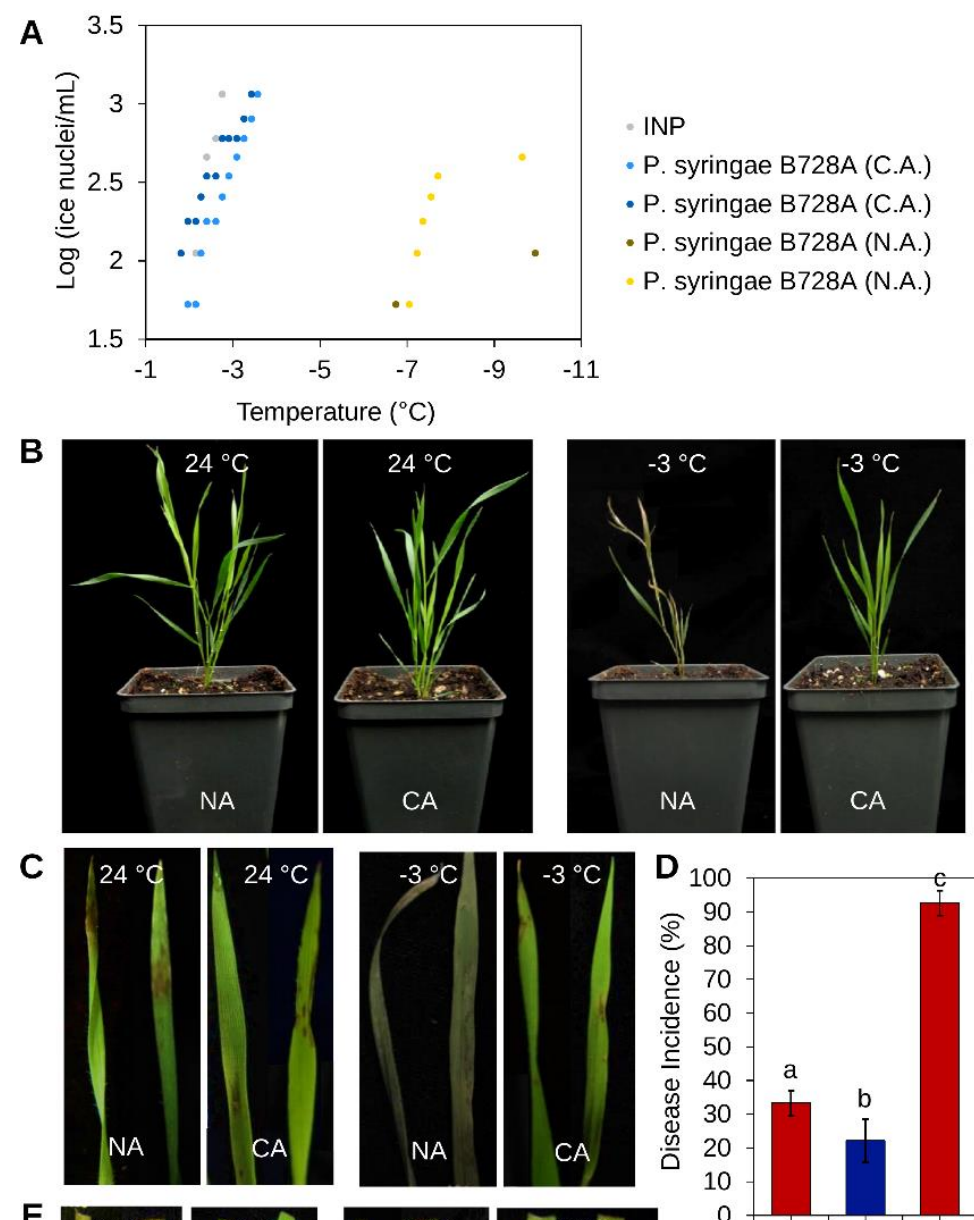

E
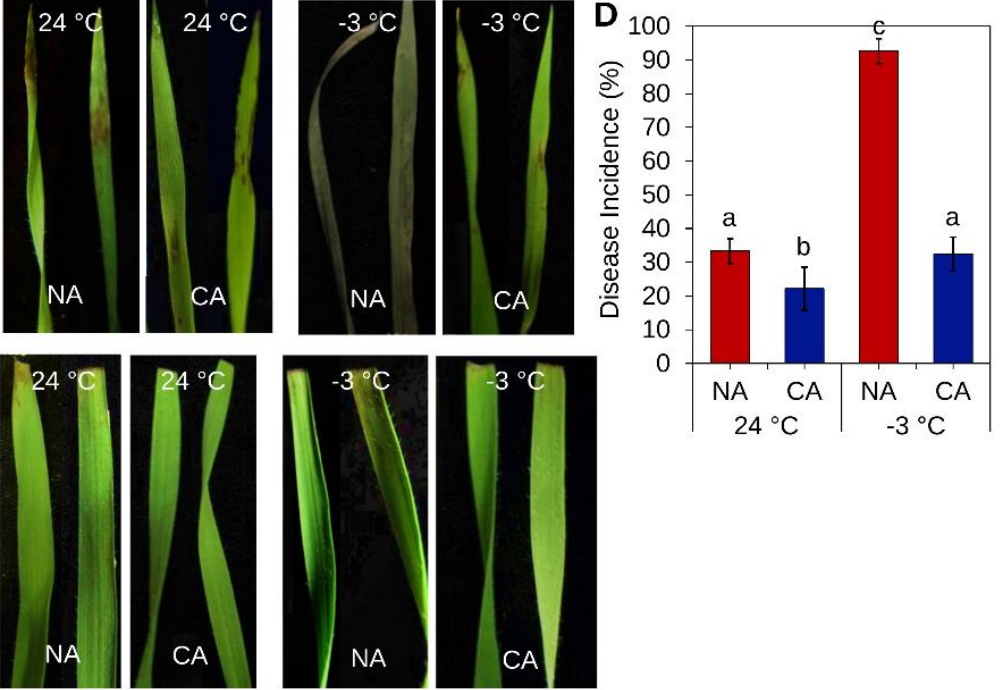

1003 Figure S9. Preliminary experiments for ice nucleation activity and infection protocols of Brachypodium distachyon with Pseudomonas syringae pv. syringae B728A. (A) Ice nucleation activity of the $P$. syringae pathovar that had been cold-activated by transfer to $4{ }^{\circ} \mathrm{C}$ for $48 \mathrm{~h}$ (C.A.) or kept at $24{ }^{\circ} \mathrm{C}$, representing non-cold-activated (N.A.) cultures with nucleation temperatures between $-2{ }^{\circ} \mathrm{C}$ and $-4{ }^{\circ} \mathrm{C}$, were used in assays to determine the conditions and temperature of incubation for leaf tissue in infection assays. Pseudomonas syringae ice nucleating protein (INP) preparations (Ward's Natural Science, Rochester, NY) were used as controls. (B) Representative non-acclimated (NA) and coldacclimated (CA) whole plants sprayed with C.A. $P$. syrinage pathovar cultures and then maintained at $24{ }^{\circ} \mathrm{C}$ (two left pots) and $-3{ }^{\circ} \mathrm{C}$ (two right pots). (C) Portions of leaves from NA and CA plants sprayed with C.A. $P$. syringae pathovar cultures at $24{ }^{\circ} \mathrm{C}$ standard conditions (the pair of left images) and -3 ${ }^{\circ} \mathrm{C}$ (the pair of right images) showing disease incidence, and in the case of the NA leaves at $-3{ }^{\circ} \mathrm{C}$, freeze damage. (D) Disease incidence measured as a percentage of leaves sprayed with the C.A. $P$. syringae pathovar showing disease symptoms with leaves from plants that were either NA and CA and then infected as whole plants and kept at the two temperatures shown. (E) Leaves taken from NA or CA plants and cut with the wounded ends dipped in cold activated $P$. syringae pathovar culture, with the excised leaves then maintained at $24{ }^{\circ} \mathrm{C}$ (left pair of images) and $-3{ }^{\circ} \mathrm{C}$ (right pair of images). 

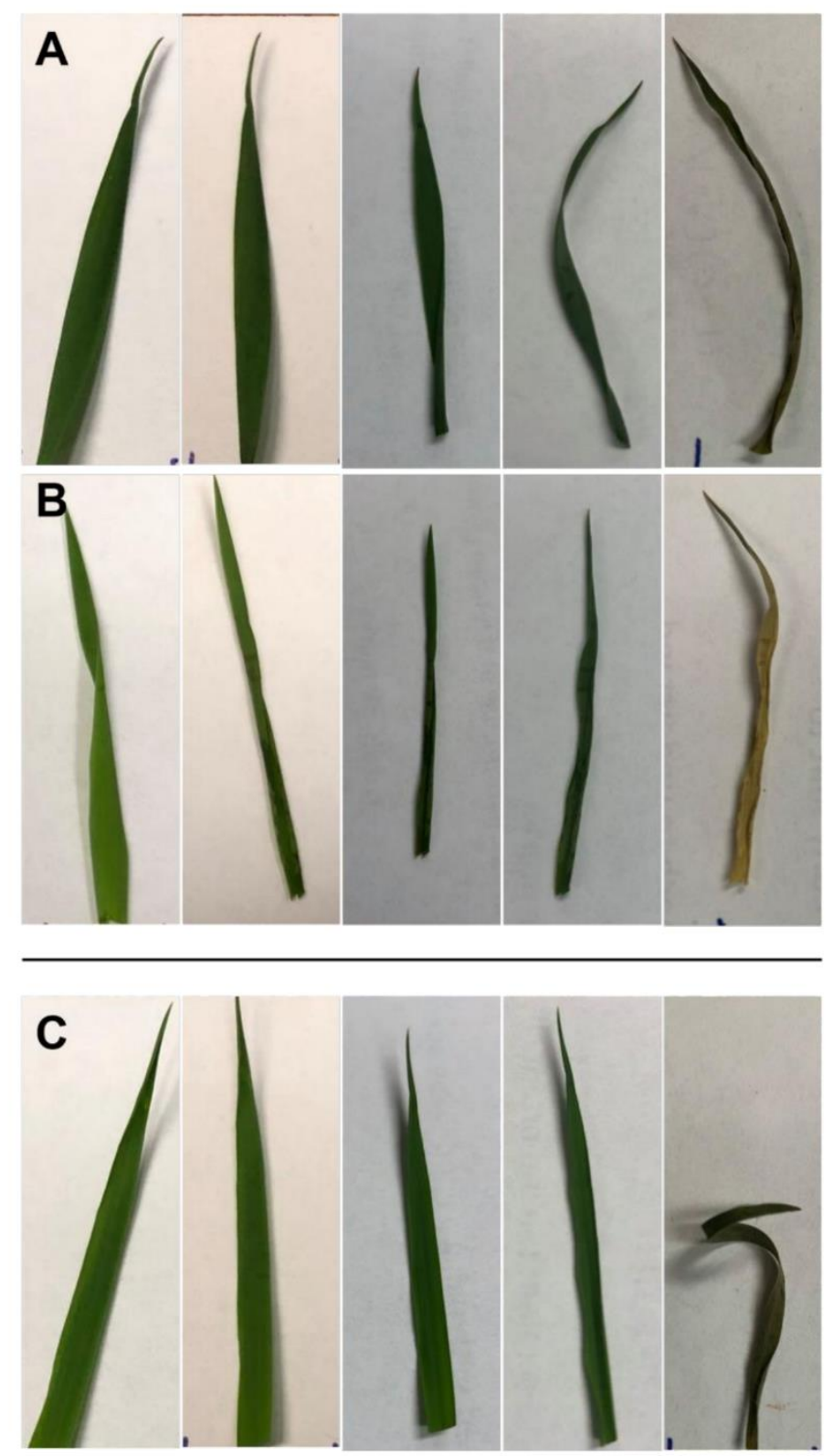

1020 Figure S10. Representative images of excised Brachypodium distachyon leaves infected with cold activated Pseudomonas syringae pv. syringae B728A, with the five images (left to right) representing pre-infection, following infection and incubation for $12 \mathrm{~h}$ at $-3{ }^{\circ} \mathrm{C}$, and during recovery at $4{ }^{\circ} \mathrm{C}$ at $24 \mathrm{~h}$ post infection, $48 \mathrm{~h}$ post infection, and one week post infection. Samples shown included: (A) infected cold-acclimated (CA) Bd21 wild type. (B) infected CA temporal cold-induced antifreeze protein (AFP) knockdown line prOmiRBdIRI-1e, and (C) uninfected CA Bd21 wild type controls. As indicted in the Methods section, the bacterial strain was cultured at $28{ }^{\circ} \mathrm{C}$ to $\mathrm{OD}_{600}=0.6-1.0$ and placed at $4{ }^{\circ} \mathrm{C}$ for two days before resuspending in $10 \mathrm{mM} \mathrm{MgCl}_{2}$ and diluted to $\mathrm{OD}_{600}=0.2$ to an approximate concentration of $1 \times 10^{8}$ colony forming units $\mathrm{mL}^{-1}$ prior to infection by dipping wounded ends of leaves non-acclimated plants (not shown). 